\title{
MATRIX-VALUED SPECIAL FUNCTIONS AND REPRESENTATION THEORY OF THE CONFORMAL GROUP, I: THE GENERALIZED GAMMA FUNCTION ${ }^{1}$ BY
}

\author{
KENNETH I. GROSS AND WAYNE J. HOLMAN III
}

In memory of our friend and colleague B.J. Pettis

ABSTRACT. This article examines in detail the matrix-valued gamma function

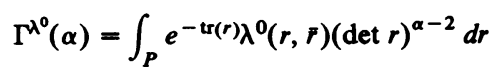

associated to the conformal group $G=U(2,2)$. Here, $\alpha$ is a continuous complex parameter, $\lambda^{0}$ runs through a family of "weights" of $K=U(2) \times U(2), P$ is the cone of $2 \times 2$ positive-definite Hermitian matrices, and the integral is well known to converge absolutely for $\operatorname{Re}(\alpha)>1$. However, until now very little has been known about the analytic continuation for the general weight $\lambda^{0}$. The results of this paper include the following: The complete analytic continuation of $\Gamma^{\lambda^{0}}$ is determined for all weights $\lambda^{0}$. In analogy to the case of the classical gamma function it is proved that for any weight $\lambda^{0}$ the mapping $\alpha \rightarrow \Gamma^{\lambda^{\circ}}(\alpha)^{-1}$ is entire. A new integral formula is given for the inverse of the gamma function. An explicit calculation is given for the normalized variant of the gamma matrix that arises in the reproducing kernel for the spaces in which the holomorphic discrete series of $G$ is realized, and one observes that the behavior of the analytic continuation for weights "in general position" is markedly different from the special cases in which the gamma function "is scalar". The full analytic continuation of the holomorphic discrete series for $G$ is determined. The gamma function for the forward light cone (the boundary orbit) is found, and the associated Hardy space of vector-valued holomorphic functions is described. Analogs are given for some of the well-known formulas for the classical gamma function. As an epilogue, applications of the matrix-valued gamma function, such as generalizations to $2 \times 2$ matrix space of the classical binomial theorem, are announced. These applications require a detailed understanding of the (generalized) Bessel functions associated to the conformal group that will be treated in the sequel to this paper.

\section{Contents}

1. Introduction

2. The conformal group and its accoutrements

3. The generalized gamma function

4. Analytic continuation of the gamma function

5. The holomorphic discrete series of representations of the conformal group

6. The normalized gamma function and analytic continuation of the holomorphic discrete series

Received by the editors July 24, 1978.

AMS (MOS) subject classifications (1970). Primary 22K45, 22E30, 33A75, 43A80; Secondary 32M15, 33A15, 81A78, 46K20.

Key words and phrases. Conformal group, matrix-valued gamma function, representation theory, holomorphic discrete series, special functions.

${ }^{1}$ This research was supported in part by National Science Foundation grant MCS73-08750.

(C) 1980 American Mathematical Society 0002-9947/80/0000-0151/\$09.00 
7. Miscellaneous properties of the gamma function

8. Proofs of the main theorems and formulas for the matrix entries

9. Concluding remarks

Appendix A: The hypergeometric function

Appendix B: Fine structure of the representations of $U(2)$

Addendum: The gamma function for $U(n, n)$

1. Introduction. This paper is the first of a series in which we shall examine in great detail the matrix-valued special functions that arise in both the harmonic analysis and the mathematical physics associated with the conformal group $U(2,2)$. Here, we are principally concerned with the generalized gamma function ((3.11) below), its intrinsic structure, its connection with the holomorphic discrete series of infinite-dimensional irreducible representations of the conformal group, the analytic continuation of the holomorphic discrete series, and the construction of Hardy-type Hilbert spaces of holomorphic functions on the corresponding Siegel upper half-plane. The key results were stated in a previous announcement [8]. In this paper we shall prove the theorems announced therein and present other properties of the gamma matrix. A second paper [9] will treat the generalized Bessel functions for the conformal group, their properties, interconnection with the gamma function, and relationship to the motion group of $U(2)$ on $2 \times 2$ complex matrix space (cf. §9).

A few general remarks concerning the conformal group are in order. First, the conformal group has been of use in physics since the early years of this century when it was demonstated that Maxwell's equations are covariant under this group. More recently the conformal group has been extensively applied in elementary particle physics. Especially relevant to the concepts in this paper are current results that have appeared in [13]. From a more abstract point of view, the conformal group is representative of a class of higher rank noncompact real reductive Lie groups for which the corresponding symmetric space has Hermitian structure. In particular, in this paper we solve a circle of related problems in analysis on the conformal group, the analogs of which remain intractible for all other such reductive Lie groups (cf., the introduction to $\$ 3$ and the conclusion of $\$ 4$ ).

The organization of this paper is as follows. In $\$ 2$ we discuss the conformal group, summarize its structure, and describe the action by linear fractional transformation on the Siegel upper half-plane. We introduce the generalized gamma function in $\$ 3$, and we bring together the elementary properties of the representation theory of $G L(2, C)$ that are needed to analyze the convergence of the integral formula for this gamma function. The complete analytic continuation of the gamma function is presented, without proof, in $\$ 4$. As a corollary, we prove that the inverse of the generalized gamma function, as in the classical case, is an entire function, and we obtain an integral representation for the inverse. $\$ \$ 5$ and 6 deal with the applications of the gamma function to the holomorphic discrete series of representations of the conformal group. More specifically, at the outset of $\$ 5$ we explain how the gamma function relates to harmonic analysis on Hermitian symmetric spaces. We then outline the construction of the Hilbert spaces of holomorphic functions in which the representations of the holomorphic discrete 
series act, and we determine the reproducing kernels for these spaces. In \$6, we give the analog of the functional equation for the gamma function and use it to define a normalized version of the gamma function. We next present without proof the explicit determination of the normalized gamma function, and apply the result to achieve the full analytic continuation of the holomorphic discrete series. We conclude $\$ 6$ with the determination of the gamma function associated to the (boundary) light cone and the corresponding Hardy-type Hilbert space. $\$ 7$ contains various properties of the gamma matrix which are analogous to well-known properties of the classical gamma function. $\$ 8$ presents the detailed proofs that were postponed from $\S \S 4$ and 6 of the formulas for the eigenvalues of the gamma matrix, the trace function, and the gamma function for the boundary. These proofs consist of intricate calculations that depend upon the explicit structure of the irreducible representations of the unitary group $U(2)$. The required representationtheoretic facts are brought together in Appendix B, and needed properties of the hypergeometric function are listed in Appendix A. Finally, §9, essentially an epilogue, takes note of some applications of the gamma function-e.g., generalizations of the binomial theorem-that will appear in the sequel [9].

This paper is arranged in such a way that the reader who is interested in the harmonic analysis on the conformal group but not the details of the calculations can omit $\S \S 7,8,9$ and the two appendices. On the other hand, the reader interested only in the special functions can omit the harmonic analysis in $\$ \S 5$ and 6.

To conclude on a personal note, it is a pleasure to acknowledge the influence of R. A. Kunze on the ideas in this paper. For example, it was he who, several years ago, first conjectured that the inverse of the gamma matrix should be entire, and that Hardy spaces for boundary components should appear in the analytic continuation of the holomorphic discrete series.

2. The conformal group and its accoutrements. Let $M=\mathrm{C}^{2 \times 2}$ denote the algebra of all $2 \times 2$ complex matrices, let $S$ be the real subspace of Hermitian matrices, let $P$ be the cone in $S$ of all positive-definite matrices, and set $H=S+i P$, the Siegel upper half-plane in $M$ of matrices $z=x+i y$ with $x=x^{*}$ and $y>0$. For $z \in M$, let $\Delta(z)=\operatorname{det}(z)$. Set $A=G L(2, \mathrm{C})$ the general linear group of all $a \in M$ such that $\Delta(a) \neq 0$, and denote by $U=U(2)$ the unitary subgroup of matrices $u$ for which $u u^{*}=u^{*} u=1$. Now, the group $A$ acts transitively (from the right) on $P$ by $r \rightarrow a^{*} r a$ for $a \in A, r \in P$; and as a homogeneous space, $P \simeq U \backslash A$. That is to say, the mapping $U a \rightarrow r=a^{*} a$ identifies the space $U \backslash A$ of right cosets $U a$ with $P$. If we fix a Lebesgue measure $d r$ on $P$, then the measure $d_{*} r=\Delta(r)^{-2} d r$ on $P$ is invariant for the action of $A$. In terms of the coordinates $x_{0}, x_{1}, x_{2}, x_{3}$ on $S$ given by

$$
x=\left(\begin{array}{ll}
x_{0}+x_{1} & x_{2}+i x_{3} \\
x_{2}-i x_{3} & x_{0}-x_{1}
\end{array}\right),
$$

$S$ is identified with $\mathbf{R}^{4}$, and the Lorentz metric is $\Delta(x)=x_{0}^{2}-x_{1}^{2}-x_{2}^{2}-x_{3}^{2}$. Equipped with the Lorentz metric, $S$ is termed flat Minkowski space and $P$ is the interior of the forward light cone. 
The conformal group $G=U(2,2)$ is defined as follows. Let us write a $4 \times 4$ complex matrix $g$ in block form

$$
g=\left(\begin{array}{ll}
g_{11} & g_{12} \\
g_{21} & g_{22}
\end{array}\right)
$$

with $g_{i j} \in M$, and let $p=\left(p_{i j}\right)$ be that $4 \times 4$ matrix such that $p_{11}=p_{22}=0$, $p_{12}=1$, and $p_{21}=-1$. Then the group $G$ consists of all $4 \times 4$ complex matrices $g$ such that $g p g^{*}=p$. Alternatively, the matrix (2.2) is in $G$ if and only if it satisfies the following three identities: $g_{11} g_{22}^{*}-g_{12} g_{21}^{*}=1, g_{11} g_{12}^{*}=g_{12} g_{11}^{*}$, and $g_{21} g_{22}^{*}=$ $g_{22} g_{21}^{*}$. For $g \in G$ and $z \in H$, the matrix $z g_{12}+g_{22}$ is invertible and the formula

$$
z \circ g=\left(z g_{12}+g_{22}\right)^{-1}\left(z g_{11}+g_{21}\right)
$$

defines an action of $G$ on $H$ by linear fractional transformations. This action of the conformal group on the Siegel upper half-plane is transitive, and as a homogeneous space $H \cong K \backslash G$, where $K$ is the stability group of the scalar matrix $i \in H$. That is, $K=\{k \in G: i \circ k=i\}$. The group $K$ can be described in terms of (2.2) as the collection of all matrices of the form

$$
k=\left(\begin{array}{ll}
k_{11} & k_{12} \\
-k_{12} & k_{11}
\end{array}\right)
$$

such that $u_{1}=k_{11}+i k_{12}$ and $u_{2}=k_{11}-i k_{12}$ are unitary. Thus, the groups $K$ and $\mathcal{K}=U \times U$ are isomorphic by means of the mapping $k \rightarrow\left(u_{1}, u_{2}\right)$. Finally, we note that the measure $d_{*} z=\Delta(y)^{-4} d x d y$ on $H$ (where $z=x+i y, x \in S, y \in P$ ) is invariant under the action (2.3) of $G$. For details, we refer to [7].

In general terminology, $G$ is a noncompact reductive Lie group (more precisely, modulo the one-dimensional center composed of scalar matrices of absolute value one, $G$ is simple), $K$ is its maximal compact subgroup, and $H$ is an unbounded realization of the Hermitian symmetric space $K \backslash G$.

3. The generalized gamma function. Let $\lambda$ be an irreducible holomorphic finite-dimensional representation of the product group $\mathbb{Q}=A \times A$. Then whenever the integral is absolutely convergent, we set

$$
\Gamma(\lambda)=\int_{P} e^{-\operatorname{tr}(r)} \lambda(r, \bar{r}) d_{*} r .
$$

This generalization of the familiar integral formula for the classical gamma function arises naturally in the infinite-dimensional harmonic analysis of the conformal group (cf., [7], [8], and \$5, below).

In this section, we use the well-known structure of the dual object $\hat{U}$ of $U=U(2)$ to describe the convergence of (3.1) and the dependence of (3.1) upon one continuous parameter and two discrete parameters. In the next section we shall calculate the full analytic continuation of this generalized gamma function. That is a more complicated problem which requires the deeper, less well-known, fine structure of the representation theory of $U$.

For each nonnegative integer $\nu$, the group $U_{0}=S U(2)=\{u \in U: \Delta(u)=1\}$ admits precisely one irreducible unitary representation $\rho_{\nu}$ (up to equivalence) of 
degree $\nu+1$. This representation extends to a polynomial representation of $A=$ $G L(2, \mathrm{C})$. Indeed, let $V_{\nu}$ denote the $(\nu+1)$-dimensional space of all polynomial functions in one variable of degree less than or equal to $\nu$. Then $\rho_{\nu}$ can be realized in the representation space $V_{\nu}$ by the formula

$$
\left(\rho_{\nu}(a) f\right)(t)=\left(a_{12} t+a_{22}\right)^{\nu} f\left(\frac{a_{11} t+a_{21}}{a_{12} t+a_{22}}\right)
$$

for $a=\left(a_{i j}\right) \in A$ and $f \in V_{\nu}$. It is standard notation [19, Chapter III] to let

$$
\nu=2 l,
$$

so $l$ is either an integer or half-integer, and to parametrize these representations by $l$. For convenience, we replace $\rho_{\nu}$ by $\rho^{l}$ and $V_{\nu}$ by $V^{l}$.

Next, consider the finite-dimensional representation theory of $\mathbb{Q}=A \times A$. Denote by $\hat{\mathscr{Q}}_{0}$ the collection of irreducible polynomial representations $\lambda^{0}=$ $\lambda^{0}\left(\cdot ; l_{1}, l_{2}\right)$ of $\mathbb{Q}$ given by

$$
\lambda^{0}\left(a_{1}, a_{2}\right)=\rho^{l_{1}}\left(a_{1}\right) \otimes \rho^{l_{2}}\left(a_{2}\right)
$$

for $\left(a_{1}, a_{2}\right) \in \mathbb{Q}$. Of course, the degree of $\lambda^{0}$ is $\left(2 l_{1}+1\right)\left(2 l_{2}+1\right)$. Note that the restrictions to the subgroup $U_{0} \times U_{0}$ of the representations $\lambda^{0}$ form a complete set of representatives for the dual object $\left(U_{0} \times U_{0}\right)^{\wedge}$. We note in passing, that for the sequel it will be necessary to have matrix-valued realizations of the representations $\lambda^{0}$ relative to two different orthogonal bases for the representation space (cf., Appendix B).

Finally, let

$$
\lambda\left(a_{1}, a_{2}\right)=\Delta\left(a_{1}\right)^{\sigma_{1}} \Delta\left(a_{2}\right)^{\sigma_{2}} \lambda^{0}\left(a_{1}, a_{2}\right)
$$

for $\left(a_{1}, a_{2}\right) \in \mathscr{Q}$, where $\lambda^{0} \in \hat{\mathfrak{Q}}_{0}$ and $\sigma_{1}, \sigma_{2}$ are integers. (3.5) defines a representation $\lambda=\lambda\left(\cdot ; \sigma_{1}, \sigma_{2}, \lambda^{9}\right)$ of $\mathcal{Q}$; and, in fact, the collection $\hat{\mathcal{Q}}$ of such representations is a complete set of irreducible holomorphic finite-dimensional representations of $\mathcal{Q}$. In the technical jargon, $\lambda$ is the tensor product of the two irreducible representations of $A$ having "highest weights" $\left(2 l_{1}+\sigma_{1}, \sigma_{1}\right)$ and $\left(2 l_{2}+\sigma_{2}, \sigma_{2}\right)$, respectively. Without loss of generality we can assume that the restriction of $\lambda$ to the subgroup $U \times U$ is unitary, or equivalently,

$$
\lambda\left(a_{1}, a_{2}\right)^{*}=\lambda\left(a_{1}^{*}, a_{2}^{*}\right)
$$

for all $\left(a_{1}, a_{2}\right) \in \mathbb{Q}$.

We now observe that formula (3.1) can be rewritten as

$$
\Gamma(\lambda)=\int_{P} e^{-\operatorname{tr}(r)} \lambda^{0}(r, \bar{r}) \Delta(r)^{\sigma_{1}+\sigma_{2}} d_{*} r .
$$

More generally, for $\lambda^{0} \in \hat{\mathbb{A}}_{0}, \alpha \in \mathbf{C}$, and $z \in H$, we set

$$
\Gamma\left(z ; \alpha, \lambda^{0}\right)=\int_{P} e^{i \operatorname{tr}(z r)} \lambda^{0}(r, \bar{r}) \Delta(r)^{\alpha} d_{*} r
$$

whenever the integral is absolutely convergent. From $\$ 3$ of [7], or directly, we can describe the convergence of (3.8) as follows. 
Theorem 3.1. Fix $\lambda^{0} \in \hat{\mathbb{Q}}_{0}$ and let $\alpha \in \mathbf{C}, z \in H$. Then the integral defining $\Gamma\left(z ; \alpha, \lambda^{9}\right)$ converges absolutely if and only if $\operatorname{Re}(\alpha)>1$. When this is the case

$$
\Gamma\left(a z a^{*} ; \alpha, \lambda^{0}\right)=|\Delta(a)|^{-2 \alpha} \lambda^{0}(a, \bar{a})^{*-1} \Gamma\left(z ; \alpha, \lambda^{0}\right) \lambda^{0}(a, \bar{a})^{-1}
$$

for all $z \in H$ and $a \in A$.

We call attention to the special case in which $z=i$ (the multiple $i$ of the identity matrix). The mapping $\alpha \rightarrow \Gamma\left(i ; \alpha, \lambda^{9}\right)$, for fixed $\lambda^{0}$, is holomorphic in the half-plane $\operatorname{Re}(\alpha)>1$. Moreover, from (3.9) with $z=i$ and $a=y^{1 / 2}$

$$
\Gamma\left(i y ; \alpha, \lambda^{0}\right)=\Delta(y)^{-\alpha} \lambda^{0}\left(y^{1 / 2}, \bar{y}^{1 / 2}\right)^{-1} \Gamma\left(i ; \alpha, \lambda^{0}\right) \lambda^{0}\left(y^{1 / 2}, \bar{y}^{1 / 2}\right)^{-1}
$$

for all $y \in P$. Thus, for fixed $\alpha$ and $\lambda^{0}$, the single operator $\Gamma\left(i ; \alpha, \lambda^{9}\right)$ completely determines the mapping $z \rightarrow \Gamma\left(z ; \alpha, \lambda^{9}\right)$ on the Siegel upper half-plane. For once $\Gamma\left(z ; \alpha, \lambda^{9}\right)$ is known for $z=i y$ (i.e., on the "imaginary axis" in $H$ ), the values for all other $z$ are determined by analytic continuation in $H$. The mapping $\alpha \rightarrow$ $\Gamma\left(i ; \alpha, \lambda^{0}\right)$ is the principal object of study in this paper. For simplicity of notation, set $\Gamma\left(i ; \alpha, \lambda^{0}\right)=\Gamma^{\lambda^{0}}(\alpha)$.

Definition 3.2. Fix $\lambda^{0} \in \hat{\mathbb{A}}_{0}$. The generalized gamma function of weight $\lambda^{0}$ for the conformal group is that operator-valued function of $\alpha$ defined by

$$
\Gamma^{\lambda^{0}}(\alpha)=\int_{P} e^{-\operatorname{tr}(r)} \lambda^{0}(r, \bar{r}) \Delta(r)^{\alpha} d_{*} r
$$

when $\operatorname{Re}(\alpha)>1$, and is defined elsewhere by analytic continuation in $\alpha$. When it becomes necessary to make explicit the dependence upon the parameters $l_{1}$ and $l_{2}$, we shall write $\Gamma\left(\alpha \mid l_{1}, l_{2}\right)$ for $\Gamma^{\lambda^{0}}(\alpha)$.

From (3.9) and (3.11), the following properties are easily established.

COROLlaRY 3.3. Let $\lambda^{0} \in \hat{\mathbb{Q}}_{0}$ and suppose $\alpha$ is a real number greater than one. Then $\Gamma^{\lambda^{0}}(\alpha)$ is a positive-definite linear transformation. Furthermore,

$$
\lambda^{0}(u, \bar{u}) \Gamma^{\lambda^{0}}(\alpha)=\Gamma^{\lambda^{0}}(\alpha) \lambda^{0}(u, \bar{u})
$$

for all $u \in U$.

As we shall see in the next section, this corollary is of crucial importance for the calculation of $\Gamma^{\lambda^{0}}(\alpha)$.

4. Analytic continuation of the gamma function. In this section we state the main theorem, which gives the complete analytic continuation in $\alpha$ of the generalized gamma function. In fact, we obtain the matrices $\Gamma^{\lambda^{0}}(\alpha)$ in diagonal form, and calculate the eigenvalues in terms of the classical gamma function.

Throughout this section, $\lambda^{0}=\lambda^{0}\left(\cdot ; l_{1}, l_{2}\right)$ is fixed, and $l$ is a parameter that takes on the values $\left|l_{1}-l_{2}\right|,\left|l_{1}-l_{2}\right|+1,\left|l_{1}-l_{2}\right|+2, \ldots, l_{1}+l_{2}$. Note that $l$ varies through integer values if $2 l_{1}+2 l_{2}$ is even, and $l$ varies through half-integer values if $2 l_{1}+2 l_{2}$ is odd. In either case $l_{1}+l_{2}-l$ is always a nonnegative integer. The symbol $\Gamma$, without appended $\lambda^{0}$ or $l_{1}$ and $l_{2}$, refers to the ordinary classical gamma function. 
THEOREM 4.1. The linear transformations $\Gamma^{\lambda^{0}}(\alpha)$, for $\operatorname{Re}(\alpha)>1$, form a commutative family of normal operators. More specifically, if $W^{\lambda^{0}}$ denotes the representation space of $\lambda^{0}$ (in notation of $\left.\S 3, W^{\lambda^{0}}=V^{l_{1}} \otimes V^{l_{2}}\right)$, then

$$
W^{\lambda^{0}}=\sum_{l=\left|l_{1}-l_{2}\right|}^{l_{1}+l_{2}} W_{l}
$$

where $W_{l}$ is an eigenspace for $\Gamma^{\lambda^{0}}(\alpha), \operatorname{dim} W_{l}=2 l+1$, and the eigenvalue of $\Gamma^{\lambda^{0}}(\alpha)$ corresponding to $W_{l}$ is

$$
\gamma_{l}^{\lambda^{0}}(\alpha)=\frac{\Gamma\left(\alpha+2 l_{1}\right) \Gamma\left(\alpha+2 l_{2}\right) \Gamma\left(\alpha+2 l_{1}+2 l_{2}+1\right) \Gamma(\alpha-1)}{\Gamma\left(\alpha+l_{1}+l_{2}-l\right) \Gamma\left(\alpha+l_{1}+l_{2}+l+1\right)} .
$$

The linear transformation $\Gamma^{\lambda^{0}}(\alpha)$ can be considered as a $d \times d$ matrix, $d=$ $\operatorname{deg} \lambda^{0}=\left(2 l_{1}+1\right)\left(2 l_{2}+1\right)$. As such, it can be brought to block diagonal form, in which the blocks are the scalar matrices $\gamma_{l}^{\lambda^{0}}(\alpha) 1_{l}, 1$, being the $(2 l+1) \times(2 l+1)$ identity matrix.

The proof, which is quite technical, is postponed to $\S 8$. However, we can give a simple conceptual explanation of the theorem.

Consider the commutation relation (3.12), and the form (3.4) of the representation $\lambda^{0}$. The representation $u \rightarrow \lambda^{0}(u, \bar{u})$ of $U$ is the tensor product of two irreducible representations of $U$; namely, $u \rightarrow \rho^{l_{1}}(u)$ and $u \rightarrow \rho^{l_{2}}(\bar{u})$. From the Clebsch-Gordan formula for the group $U$ (cf., Appendix B), one knows that such tensor products are multiplicity-free. In this case

$$
\lambda^{0}(u, \bar{u})=\sum_{l=\left|l_{1}-l_{2}\right|}^{l_{1}+l_{2}} \Delta(u)^{l_{1}-l_{2}-\rho^{l}} \rho^{l}(u)
$$

for $u \in U$. Thus, the first statement of the theorem is an elementary consequence of the fact that $\Gamma^{\lambda^{0}}(\alpha)$ commutes with a multiplicity-free representation, and the form (4.1) of the eigenspace decomposition follows from (4.3). However, the computation of the eigenvalues in the convenient form (4.2) uses the full array of explicit combinatoric and calculational formulas available for the group $U(2)$ as a result of its role in quantum mechanics.

We close this section with some elementary observations based upon (4.2).

Recall that it is a characteristic property of the classical gamma function that the multiplicative inverse $1 / \Gamma(\alpha)$ is an entire function of the complex variable $\alpha$. Now, from Corollary 3.3 we know that $\Gamma^{\lambda^{0}}(\alpha)^{-1}$ exists (in fact, it is positive-definite) for real numbers $\alpha>1$. Consequently, we can ask whether an analogue of the above classical property exists for $\Gamma^{\lambda^{0}}$. The answer is in the affirmative, and follows easily from (4.2).

COROllaRY 4.2. For $\operatorname{Re}(\alpha)>1$, the linear transformation $\Gamma^{\lambda^{0}}(\alpha)$ is invertible, and the mapping $\alpha \rightarrow \Gamma^{\lambda^{0}}(\alpha)^{-1}$ has an analytic continuation to an entire function of $\alpha$.

Proof. Interchange numerator and denominator in (4.2). Note first that $l_{1}+l_{2}$ $-l$ is a nonnegative integer; so if $\Gamma\left(\alpha+l_{1}+l_{2}-l\right)$ has a pole (as a function of $\alpha)$, then so does $\Gamma(\alpha-1)$. Next, since $l>\left|l_{1}-l_{2}\right|$, it follows that $l_{1}+l_{2}+l+1$ is a nonnegative integer which is greater than either $2 l_{1}$ or $2 l_{2}$. Consequently, if 
$\Gamma\left(\alpha+l_{1}+l_{2}+l+1\right)$ has a pole, then so do both $\Gamma\left(\alpha+2 l_{1}\right)$ and $\Gamma\left(\alpha+2 l_{2}\right)$. We conclude that each of the eigenvalues $\gamma_{l}^{\lambda^{0}}(\alpha)^{-1}$ is an entire function of $\alpha$, and the corollary is proved.

There are two special cases in which the gamma function is particularly simple (cf. [5], [13]).

COROLlaRy 4.3. The gamma function $\Gamma^{\lambda^{0}}$ is scalar-valued if and only if either $l_{1}=0$ or $l_{2}=0$. Specifically,

$$
\Gamma\left(\alpha \mid l_{1}, 0\right)=\Gamma(\alpha-1) \Gamma\left(\alpha+2 l_{1}\right) 1
$$

and

$$
\Gamma\left(\alpha \mid 0, l_{2}\right)=\Gamma(\alpha-1) \Gamma\left(\alpha+2 l_{2}\right) 1
$$

where 1 denotes the identity transformation on $W^{\lambda^{0}}$.

Proof. By (3.4) and (4.1), the representation $u \rightarrow \lambda^{0}(u, \bar{u})$ is irreducible if and only if either $l_{1}$ or $l_{2}$ vanishes. Formulas (4.4) and (4.5) are special cases of (4.2).

In the next section we apply Theorem 4.1 to the representation theory of the conformal group. As we shall see, the special cases in which the gamma function is scalar (Corollary 4.3) are distinctly different from the general case.

We close this section with an analysis of the denominator in the eigenvalue (4.2), and a resulting integral formula for the inverse gamma function.

On a priori grounds, it might appear more natural to delete the complex conjugate in the definition (3.11) of the gamma function and define a more "symmetrical" gamma function

$$
\tilde{\Gamma}^{\lambda^{0}}(\alpha)=\int_{P} e^{-\operatorname{tr}(r)} \lambda^{0}(r, r) \Delta(r)^{\alpha} d_{*} r
$$

As before, the integral converges for $\operatorname{Re}(\alpha)>1$.

In analogy to Corollary $3.3, \tilde{\Gamma}^{\lambda^{0}}(\alpha)$ commutes with the representation $u \rightarrow$ $\lambda^{0}(u, u)$ of $U$, and a theorem corresponding to Theorem 4.1 holds for this new gamma function. In this case, the eigenvalues $\tilde{\gamma}_{l}^{\lambda^{0}}(\alpha)$ of $\tilde{\Gamma}^{\lambda^{0}}(\alpha)$ are more simply given by the formula

$$
\tilde{\gamma}_{l}^{\lambda^{0}}(\alpha)=\Gamma\left(\alpha+l_{1}+l_{2}-l-1\right) \Gamma\left(\alpha+l_{1}+l_{2}+l\right) .
$$

[In point of fact, the calculation (4.7) for $\tilde{\Gamma}^{\lambda^{0}}$ is rather easy to achieve, whereas formula (4.2) for $\Gamma^{\lambda^{0}}$ is much more intricate. In particular, (4.7) involves nothing more than the most elementary properties of the Clebsch-Gordan (or Wigner) coefficients that "couple" two irreducible representations of $S U(2)$; but (4.2) requires the Racah coefficients for the "recoupling" of three such representations. Cf., Appendix B.] As we indicate in \$8, (4.7) follows immediately from (8.10).

Comparison of (4.7) and (4.2) reveals that the new gamma function $\tilde{\Gamma}^{\lambda^{0}}$ is intimately related to the inverse of $\Gamma^{\lambda^{0}}$. To make this relationship precise, call the numerator in (4.2) by the symbol $\xi$. That is,

$$
\xi^{\lambda^{0}}(\alpha)=\Gamma\left(\alpha+2 l_{1}\right) \Gamma\left(\alpha+2 l_{2}\right) \Gamma\left(\alpha+2 l_{1}+2 l_{2}+1\right) \Gamma(\alpha-1)
$$

for all $\alpha$ and $\lambda^{0}$. Set

$$
\tilde{\lambda}^{0}\left(a_{1}, a_{2}\right)=\lambda^{0}\left(a_{1}, p a_{2} p^{-1}\right)
$$


for $a_{1}, a_{2} \in A$ (cf., the beginning of $\S 8$ ). Clearly, $\tilde{\lambda}^{0}$ and $\lambda^{0}$ are equivalent. We then see that $\Gamma^{\lambda^{0}}(\alpha)$ and $\tilde{\Gamma}^{\tilde{\lambda}^{0}}(\alpha)$, as defined by (3.11), with (3.4), and (4.6) and (4.9), can be diagonalized simultaneously. They commute with one another, and

$$
\Gamma^{\lambda^{0}}(\alpha) \tilde{\Gamma}^{\tilde{\lambda}^{0}}(\alpha+1)=\xi^{\lambda^{0}}(\alpha) I
$$

where $I$ denotes the identity transformation. Equivalently,

$$
\Gamma^{\lambda^{0}}(\alpha)^{-1}=\left(\frac{1}{\xi^{\lambda^{0}}(\alpha)}\right) \tilde{\Gamma}^{\tilde{\lambda}^{0}}(\alpha+1) ;
$$

or as an integral formula for the inverse gamma function

$$
\Gamma^{\lambda^{0}}(\alpha)^{-1}=\left(\frac{1}{\xi^{\lambda^{0}}(\alpha)}\right) \int_{P} e^{-\operatorname{tr}(r)} \tilde{\lambda}^{0}(r, r) \Delta(r)^{\alpha+1} d_{*} r,
$$

the integral being convergent for $\operatorname{Re}(\alpha)>0$. Symbolically, we can rewrite (4.2) in the basis-free form

$$
\Gamma^{\lambda^{0}}(\alpha)=\xi^{\lambda^{0}}(\alpha) / \tilde{\Gamma}^{\tilde{\lambda}^{0}}(\alpha+1)
$$

5. The holomorphic discrete series of representations of the conformal group. In this section we give a short sketch of the realization of the holomorphic discrete series of the conformal group in which the generalized gamma function (3.11) plays a central role. Details for this construction appear in [7].

We begin with a few brief remarks concerning the general context. A noncompact semisimple, or reductive, Lie group for which the symmetric space $G / K$ is Hermitian admits a family of infinite-dimensional irreducible unitary representations known as the holomorphic discrete series. These representations were originally constructed by Harish-Chandra [11]. The adjective "discrete" refers to the fact that these representations appear discretely in the Plancherel formula for G. Equivalently, the matrix entries of these representations are square-integrable over $G$ (or over $G$ modulo the center, in the event that the center of $G$ is noncompact). The term "holomorphic" refers to the fact that these representations can be realized in Hilbert spaces of holomorphic functions on $G / K$. If the symmetric space is realized in unbounded form as a so-called Siegel domain, and if the holomorphic functions are chosen to be vector-valued, then the generalized gamma function arises naturally in the construction of the holomorphic discrete series and in applications in number theory and analysis [3]-[7], [13], [16], [17].

Let us restrict our attention to the conformal group $G=U(2,2)$. The appropriate Hilbert spaces of holomorphic functions on the Siegel upper half-plane $H$ will be described in two different ways: First, explicitly in terms of an inner product that is derived from an operator-valued measure; and second, by means of a positive-definite reproducing kernel.

I. The spaces $\mathfrak{V}_{\lambda^{0}, \alpha}$. Fix $\lambda^{0} \in \hat{\mathscr{C}}_{0}$, let $\alpha$ be real, and denote by $\mathfrak{Y}_{\lambda^{0}, \alpha}$ the collection of all holomorphic functions $F: H \rightarrow W^{\lambda^{0}}$ such that

$$
\int_{H}\left(\lambda^{0}(y, \bar{y}) F(z) \mid F(z)\right) \Delta(y)^{\alpha} d_{*} z<\infty
$$


where $z=x+i y$ and $d_{*} z$ is $G$-invariant measure on $H$. Relative to the inner product

$$
\left\langle F_{1} \mid F_{2}\right\rangle_{\lambda^{0}, \alpha}=\int_{H}\left(\lambda^{0}(y, \bar{y}) F_{1}(z) \mid F_{2}(z)\right) \Delta(y)^{\alpha} d_{*} z
$$

$\mathfrak{Y}_{\lambda^{0}, \alpha}$ is a Hilbert space. The problem is to find those values of $\alpha$ for which $\mathfrak{Y}_{\lambda^{0}, \alpha}$ is nonzero.

To that end, consider the collection $L^{2}\left(P, \lambda^{0}, \alpha\right)$ of Baire functions $f: P \rightarrow W^{\lambda^{0}}$ such that

$$
\int_{P}\left\|\lambda^{0}(r, \bar{r})^{-1 / 2} f(r)\right\|^{2} \Delta(r)^{2-\alpha} d r<\infty .
$$

From (3.10), $L^{2}\left(P, \lambda^{0}, \alpha\right)$ is a Hilbert space with respect to the inner product

$$
\left\langle f_{1} \mid f_{2}\right\rangle^{\lambda^{0}, \alpha}=\int_{P}\left(\Gamma\left(2 i r ; \alpha-2, \lambda^{0}\right) f_{1}(r) \mid f_{2}(r)\right) d r .
$$

Then the Laplace transform $\mathcal{E}: f \rightarrow F$, defined by

$$
F(z)=(2 \pi)^{-2} \int_{P} e^{i \operatorname{tr}(z r)} f(r) d r
$$

for $z \in H$, is a unitary mapping of $L^{2}\left(P, \lambda^{0}, \alpha\right)$ onto $\mathfrak{V}_{\lambda^{0}, \alpha}$; i.e.,

$$
\left\langle f_{1} \mid f_{2}\right\rangle^{\lambda^{0}, \alpha}=\left\langle F_{1} \mid F_{2}\right\rangle_{\lambda^{0}, \alpha}
$$

for all $f_{1}, f_{2} \in L^{2}\left(P, \lambda^{0}, \alpha\right)$. Now, by (3.10) we see that $L^{2}\left(P, \lambda^{0}, \alpha\right)$ is nonvanishing if and only if the integral (3.11) for $\Gamma^{\lambda^{0}}(\alpha-2)$ converges absolutely. Theorem 3.1 then implies the following result, which is a variant for the conformal group of a theorem first proved by Harish-Chandra.

TheOREM 5.1. Let $\lambda^{0} \in \hat{\mathbb{Q}}_{0}$ and let $\alpha$ be real. Then $\mathfrak{Y}_{\lambda^{0}, \alpha}$ is nonvanishing if and only if $\alpha>3$.

The representations of $G$ in the holomorphic discrete series are realized in the spaces $\mathfrak{Y}_{\lambda^{0}, \alpha}$. Indeed, if $\lambda=\lambda\left(\cdot ; \sigma_{1}, \sigma_{2}, \lambda^{9}\right)$ is the representation of $\mathbb{Q}=A \times A$ given by (3.5), and if $\alpha=\sigma_{1}+\sigma_{2}>3$, then the formula

$$
(T(g, \lambda) F)(z)=\lambda\left(\left(z^{*} g_{12}+g_{22}\right)^{*-1},\left(z g_{12}+g_{22}\right)^{t-1}\right) F(z \circ g)
$$

for $g \in G=U(2,2)$ and $F \in \mathfrak{V}_{\lambda^{0}, \alpha}$, defines an irreducible unitary representation of $G$ in the space $\mathfrak{V}_{\lambda^{0}, \alpha}$. These representations $T(\cdot, \lambda)$, parametrized by $\lambda^{0}=\lambda^{0}\left(\cdot ; l_{1}, l_{2}\right)$ $\in \hat{\mathscr{Q}}_{0}$ and integers $\sigma_{1}$ and $\sigma_{2}$ such that $\alpha=\sigma_{1}+\sigma_{2}>3$, form the holomorphic discrete series of $G$.

Formula (5.7) for a representation of $G$ can be given a meaning even when $\sigma_{1}$ and $\sigma_{2}$ are not integral. In fact, if $\sigma_{1}$ and $\sigma_{2}$ are real numbers but not necessarily integers, then (3.5) defines a cocycle, or projective, representation $\lambda$ of $\mathcal{Q}$, which extends uniquely to a representation $\tilde{\lambda}=\tilde{\lambda}\left(\cdot ; \sigma_{1}, \sigma_{2}, \lambda^{9}\right)$ of the universal covering group of $\mathcal{Q}$. Then formula (5.7) defines a cocycle representation $T(\cdot, \lambda)$ of $G$ which extends uniquely to an irreducible unitary representation $T(\cdot, \tilde{\lambda})$ of the universal covering group of $G$. These representations, indexed by $\lambda^{0} \in \hat{\mathbb{C}}_{0}$ and real numbers 
$\sigma_{1}$ and $\sigma_{2}$ such that $\alpha=\sigma_{1}+\sigma_{2}>3$, are said to constitute the relative holomorphic discrete series of the universal covering group of $G$.

II. The reproducing kernel for $\mathfrak{Y}_{\lambda^{0}, \alpha}$. Let $W$ be any finite-dimensional complex Hilbert space, denote by $\mathcal{L}$ the algebra of linear transformations on $W$, and let $H$ as usual be the Siegel upper half-plane. A continuous function $Q: H \times H \rightarrow \mathcal{L}$ is called positive-definite if the following two properties hold:

$$
Q(z, z)>0
$$

(i.e., $Q(z, z)$ is positive-definite) for all $z \in H$, and

$$
\sum_{j=1}^{n}\left(Q\left(z_{j}, z_{k}\right) \phi_{k} \mid \phi_{j}\right)>0
$$

for all $z_{1}, \ldots, z_{n}$ in $H, \phi_{1}, \ldots, \phi_{n}$ in $W$, and positive integer $n$.

Each positive-definite function $Q$ gives rise to a unique Hilbert space $\mathfrak{V}=\mathfrak{V}_{Q}$ of continuous $W$-valued functions on $H$. Conversely, if $\mathfrak{V}$ is a Hilbert space of continuous $W$-valued functions on $H$ in which point evaluations are continuous, then there exists a positive-definite function $Q$, the reproducing kernel for $\mathfrak{Y}$, such that $\mathfrak{Y}=\mathfrak{Y}_{Q}$. For given $Q$, let $\mathfrak{Y}_{0}$ be the linear span of the functions $F_{w \phi}$ on $\boldsymbol{H}$ defined by $F_{w \phi}(z)=Q(z, w) \phi$, where $w \in H$ and $\phi \in W$. Then the formula

$$
\left\langle F_{w_{1} \phi_{1}} \mid F_{w_{2} \phi_{2}}\right\rangle=\left(Q\left(w_{2}, w_{1}\right) \phi_{1} \mid \phi_{2}\right)
$$

extends to an inner product on $\mathfrak{Y}_{0}$, and $\mathfrak{Y}=\mathfrak{V}_{Q}$ is the completion of $\mathfrak{Y}_{0}$ in this inner product. On the other hand, if $\mathfrak{Y}$ is a Hilbert space of continuous functions $F$ : $H \rightarrow W$ such that the evaluation mappings $F \rightarrow F(z)$ are continuous, then there is a reproducing kernel $Q$ for $\mathfrak{V}$ defined by the equation

$$
(F(w) \mid \phi)=\langle F \mid Q(\cdot, w) \phi\rangle
$$

for all $F \in \mathfrak{Y}, w \in H$, and $\phi \in W$. This kernel $Q$ is positive-definite and $\mathfrak{Y}=\mathfrak{V}_{Q}$.

Let us specialize the preceding discussion to the case in which $\lambda^{0} \in \hat{\mathbb{C}}_{0}, W=$ $W^{\lambda^{0}}$ is the representation space of $\lambda^{0}, \mathfrak{Y}=\mathfrak{Y}_{\lambda^{0}, \alpha}$ with $\alpha>3$, and $Q=Q_{\lambda^{0}, \alpha}$ is the reproducing kernel for $\mathfrak{Y}_{\lambda^{0}, \alpha}$. From the form of the inner products (5.2) and (5.4), their equivalence as expressed by (5.6), and the defining equation (5.11) for the reproducing kernel, it follows that

$$
Q_{\lambda^{0}, \alpha}(z, w)=(2 \pi)^{-4} \int_{P} e^{i \operatorname{tr}\left(z-w^{*}\right) r} \Gamma\left(2 i r ; \alpha-2, \lambda^{0}\right)^{-1} d r
$$

Alternatively, by (3.10) and (3.9), the above formula can be replaced by

$$
Q_{\lambda^{0}, \alpha}(z, w)=c\left(\lambda^{0}, \alpha\right) \int_{P} e^{i \operatorname{tr}\left(z-w^{*}\right) r} \lambda^{0}(r, \bar{r})^{1 / 2} \Gamma^{\lambda^{0}}(\alpha-2)^{-1} \lambda^{0}(r, \bar{r})^{1 / 2} \Delta(r)^{\alpha} d_{*} r
$$

for $z, w \in H$ and $\alpha>3$, where $c\left(\lambda^{0}, \alpha\right)$ is the positive constant

$$
c\left(\lambda^{0}, \alpha\right)=(2 \pi)^{-4} 4^{\alpha+l_{1}+l_{2}-2} \text {. }
$$

By means of an argument that leans heavily upon the representation $T(\cdot, \tilde{\lambda})$, one

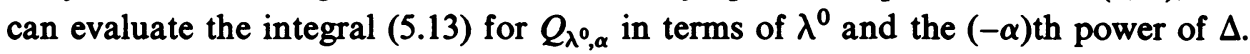


Indeed,

$$
\begin{aligned}
\int_{P} e^{i \operatorname{tr}(z r)} \lambda^{0}(r, \bar{r})^{1 / 2} \Gamma^{\lambda^{0}}(\alpha-2)^{-1} \lambda^{0}(r, \bar{r})^{1 / 2} \Delta(r)^{\alpha} d_{*} r \\
=d_{\lambda}^{-1} \operatorname{tr}\left(\Gamma^{\lambda^{0}}(\alpha-2)^{-1} \Gamma^{\lambda^{0}}(\alpha)\right) \Delta(-i z)^{-\alpha} \lambda^{0}\left(-i z,-i z^{t}\right)^{-1}
\end{aligned}
$$

for $z \in H$, where $d_{\lambda}=\operatorname{deg} \lambda^{0}$. Consequently,

$$
\begin{aligned}
Q_{\lambda^{0}, \alpha}(z, w)= & d_{\lambda}^{-1} c\left(\lambda^{0}, \alpha\right) \operatorname{tr}\left(\Gamma^{\lambda^{0}}(\alpha-2)^{-1} \Gamma^{\lambda^{0}}(\alpha)\right) \\
& \times \Delta\left(-i\left(z-w^{*}\right)\right)^{-\alpha} \lambda^{0}\left(-i\left(z-w^{*}\right),-i\left(z-w^{*}\right)^{t}\right)^{-1} .
\end{aligned}
$$

One closing observation is highly important. Although we have a restriction $\alpha>3$ for the nonvanishing of the Hilbert spaces $\mathfrak{V}_{\lambda^{0}, \alpha}$, the integral formula (5.13) for the reproducing kernel $Q_{\lambda^{0}, \alpha}$ exists for $\alpha>1$. This leads us to ask the following question: For what values of $\alpha$ in the interval $1<\alpha<3$ does $Q_{\lambda^{0}, \alpha}$ remain positive-definite? This is the problem of the extent of analytic continuation of the holomorphic discrete series.

6. The normalized gamma function and analytic continuation of the holomorphic discrete series. In this section we perform the complete analytic continuation of the holomorphic discrete series. Our technique makes use of the trace that appears on the right side of equation (5.15) as a convenient normalization factor. This trace function also provides an analog for $\Gamma^{\lambda^{0}}$ of the functional equation $\Gamma(\alpha-1)^{-1} \Gamma(\alpha)$ $=\alpha-1$ for the ordinary gamma function.

Definition 6.1. For $\lambda^{0} \in \hat{\mathbb{Q}}_{0}$ and $\alpha \in \mathbf{C}$, set

$$
\psi^{\lambda^{0}}(\alpha)=d_{\lambda}^{-1} \operatorname{tr}\left(\Gamma^{\lambda^{0}}(\alpha)^{-1} \Gamma^{\lambda^{0}}(\alpha+2)\right)
$$

where $d_{\lambda}=\operatorname{deg} \lambda^{0}=\left(2 l_{1}+1\right)\left(2 l_{2}+1\right)$, and let

$$
N^{\lambda^{0}}(\alpha)=\psi^{\lambda^{0}}(\alpha-2) \Gamma^{\lambda^{0}}(\alpha-2) \text {. }
$$

We shall refer to $\psi^{\lambda^{0}}$ as the trace function, and we shall call $N^{\lambda^{0}}$ the normalized gamma function of weight $\lambda^{0}$.

In $\$ 8$ we carry out the calculation of $\psi^{\lambda^{0}}$. The result is as follows.

TheOREM 6.2. For $\lambda^{0}=\lambda^{0}\left(\cdot ; l_{1}, l_{2}\right) \in \hat{\mathbb{Q}}_{0}$ and $\alpha \in \mathbf{C}$, the trace function is the polynomial in $\alpha$ of degree four given by

$$
\psi^{\lambda^{0}}(\alpha)=\left(\alpha+2 l_{1}+2 l_{2}+1\right)\left(\alpha+2 l_{1}\right)\left(\alpha+2 l_{2}\right)(\alpha-1) .
$$

This theorem yields the functional equation $\xi^{\lambda^{0}}(\alpha+1)=\psi^{\lambda^{0}}(\alpha) \xi^{\lambda^{0}}(\alpha)$ for the function $\xi^{\lambda^{0}}$ given by (4.8). By (6.2) and (4.12) the normalized gamma function is given by $N^{\lambda^{0}}(\alpha)=\xi^{\lambda^{0}}(\alpha-1) / \tilde{\Gamma}^{\tilde{\lambda}^{0}}(\alpha-1)$. Thus:

COROllary 6.3. The subspaces $W_{l}$ of $W^{\lambda^{0}}$, as in Theorem 4.1, are eigenspaces for $N^{\lambda^{0}}(\alpha)$. The eigenvalue of $N^{\lambda^{0}}(\alpha)$ on the space $W_{l}$ is

$$
n_{l}^{\lambda^{0}}(\alpha)=\frac{\Gamma\left(\alpha+2 l_{1}-1\right) \Gamma\left(\alpha+2 l_{2}-1\right) \Gamma\left(\alpha+2 l_{1}+2 l_{2}\right) \Gamma(\alpha-2)}{\Gamma\left(\alpha+l_{1}+l_{2}-l-2\right) \Gamma\left(\alpha+l_{1}+l_{2}+l-1\right)} .
$$


COROLlaRY 6.4. (i) If either $l_{1}=0$ or $l_{2}=0$, in which case $\Gamma^{\lambda^{0}}(\alpha)$ is scalar, then $N^{\lambda^{0}}(\alpha)=\Gamma^{\lambda^{0}}(\alpha)$ for all $\alpha$. In this case, there is no distinction between normalized and unnormalized gamma functions.

(ii) If $l_{1}$ and $l_{2}$ are both nonzero, then the linear transformation $N^{\lambda^{0}}(\alpha)^{-1}$ exists for all $\alpha$ and defines an entire function of $\alpha$. Moreover, $N^{\lambda^{0}}(\alpha)^{-1}$ is positive-definite for $\alpha>2$, and $N^{\lambda^{0}}(2)^{-1}$ is positive semidefinite and of rank one. Indeed, the eigenvalue $n_{l_{1}+l_{2}}^{\lambda^{0}}(\alpha)^{-1}$ is positive when $\alpha=2$; but for $l<l_{1}+l_{2}$ the eigenvalues $n_{l}^{\lambda^{0}}(\alpha)^{-1}$ are all zero and change sign at $\alpha=2$.

Proof. Part (i) follows immediately from Corollary 4.3 and the definition (6.3) of $N^{\lambda^{0}}$. As for part (ii), the proof that the function $\alpha \rightarrow N^{\lambda^{0}}(\alpha)^{-1}$ is entire is completely analogous to the proof of Corollary 4.2. When $\alpha>2$, all of the arguments are positive in the gamma functions on the right side of (6.4), so $N^{\lambda^{0}}(\alpha)^{-1}$ is positive-definite for $\alpha>2$. Finally, we examine the behavior at $\alpha=2$. When $l=l_{1}+l_{2}$ in (6.4), we obtain

$$
n_{l_{1}+l_{2}}^{\lambda^{0}}(\alpha)=\left(\alpha+2 l_{1}+2 l_{2}-1\right) \Gamma\left(\alpha+2 l_{1}-1\right) \Gamma\left(\alpha+2 l_{2}-1\right) \text {, }
$$

and it follows that

$$
\lim _{\alpha \rightarrow 2} n_{l_{1}+l_{2}}^{\lambda^{0}}(\alpha)=n_{l_{1}+l_{2}}^{\lambda^{0}}(2)=\left(2 l_{1}+2 l_{2}+1\right) \Gamma\left(2 l_{1}+1\right) \Gamma\left(2 l_{2}+1\right)>0 .
$$

However, if $l<l_{1}+l_{2}$ then

$$
\lim _{\alpha \rightarrow 2} n_{l}^{\lambda^{0}}(\alpha)^{-1}=n_{l}^{\lambda^{0}}(2)^{-1}=\frac{\Gamma\left(l_{1}+l_{2}-l\right)}{\Gamma\left(2 l_{1}+1\right) \Gamma\left(2 l_{2}+1\right)\left(2 l_{1}+2 l_{2}+1\right) \Gamma(0)} .
$$

The presence of $\Gamma(0)$ in the denominator implies that $n_{l}^{\lambda^{0}}(2)^{-1}=0$ and that $n_{l}^{\lambda^{0}}(\alpha)^{-1}$ changes sign as $\alpha$ passes through the value 2 .

From Corollary 6.4 we easily derive the analytic continuation of the holomorphic discrete series.

In view of (5.13)-(5.16), we are led to a normalized kernel function $\mathscr{2}_{\lambda^{0}, \alpha}$ for the Hilbert space $\mathfrak{V}_{\lambda^{0}, \alpha}$, defined by

$$
2_{\lambda^{0}, \alpha}(z, w)=c\left(\lambda^{0}, \alpha\right)^{-1} \psi^{\lambda^{0}}(\alpha-2)^{-1} Q_{\lambda^{0}, \alpha}(z, w)
$$

for $z, w \in H$. Then (5.15) and (5.16) can be reformulated as

$$
\mathscr{Q}_{\lambda^{0}, \alpha}(z, w)=\int_{P} e^{i \operatorname{tr}\left(z-w^{*}\right) r} \lambda^{0}(r, \bar{r})^{1 / 2} N^{\lambda^{0}}(\alpha)^{-1} \lambda^{0}(r, \bar{r})^{1 / 2} \Delta(r)^{\alpha} d_{*} r
$$

and

$$
\mathcal{Q}_{\lambda^{0}, \alpha}(z, w)=\Delta\left(-i\left(z-w^{*}\right)\right)^{-\alpha} \lambda^{0}\left(-i\left(z-w^{*}\right),-i\left(z-w^{*}\right)^{t}\right)^{-1},
$$

respectively.

Notice that the integral representation (6.6) for the reproducing kernal $\mathscr{2}_{\lambda^{0}, \alpha}$, originally defined for $\alpha>3$, remains positive-definite for those values of $\alpha>1$ such that the linear transformation $N^{\lambda^{\circ}}(\alpha)$ is positive-definite. Thus, for these additional values of $\alpha, 2_{\lambda^{0}, \alpha}$ is a reproducing kernel for a Hilbert space, again denoted by $\mathfrak{Y}_{\lambda^{0}, \alpha}$, of holomorphic functions on $H$. Furthermore, if $N^{\lambda^{0}}(\alpha)^{-1}$ is only positive semidefinite, then $\mathscr{2}_{\lambda^{0}, \alpha}$ still corresponds to a Hilbert space $\mathfrak{Y}_{\lambda^{0}, \alpha}$ of 
holomorphic functions on $H$. (In the latter context, we must allow $Q(z, z)>0$ in (5.8), and the space $\mathfrak{Y}_{Q}$ spanned by the functions $Q(\cdot, w) \phi$ admits nonzero null vectors which must be factored out to obtain a Hilbert space structure.) The representations $T(\cdot, \tilde{\lambda})$ of the universal covering group of $G$, defined as before by (5.7), in these new Hilbert spaces $\mathfrak{V}_{\lambda^{0}, \alpha}($ where $\alpha \leqslant 3)$ are said to be in the analytic continuation of the holomorphic discrete series.

Following the terminology introduced in [17], we shall define the Wallach set for the conformal group to be the collection of all pairs $\left(\lambda^{0}, \alpha\right)$ in $\hat{\mathscr{C}}_{0} \times \mathbf{C}$ for which the function of $z$ and $w$ on the right side of (6.7) is positive-definite (or positive semidefinite; cf., the preceding paragraph).

TheOREM 6.5. Let $\lambda^{0} \in \hat{\mathbb{Q}}_{0}$.

(1) If either $l_{1}=0$ or $l_{2}=0$ (so that the gamma function is scalar), then $\left(\lambda^{0}, \alpha\right)$ is in the Wallach set for each $\alpha>1$.

(2) If both $l_{1} \neq 0$ and $l_{2} \neq 0$ (the general case), then $\left(\lambda^{0}, \alpha\right)$ is in the Wallach set for each $\alpha>2$, but $\left(\lambda^{0}, \alpha\right)$ fails to be in the Wallach set for $1<\alpha<2$.

In light of the preceding remarks, Theorem 6.5 is a restatement of Corollary 6.4 which expresses the complete analytic continuation of the holomorphic discrete series.

At this point, citations of related literature are in order. The term "Wallach set" refers to the work of $\mathrm{N}$. Wallach [20] which shows that for the general semisimple group $G$ (for which $G / K$ is Hermitian) the holomorphic discrete series admits an analytic continuation. For $G=U(n, n)$, the analytic continuation was constructed by Gross and Kunze [5] for that portion of the holomorphic discrete series for which the gamma function is scalar. In [17], Rossi and Vergne calculated, for general $G$, that portion of the Wallach set corresponding to $\lambda^{0}=1$, the identity representation. They also showed that the "integer points" in the range of analytic continuation correspond to Hardy spaces associated with the boundary components of the symmetric space. For $G=U(2,2)$, their results correspond to the case $l_{1}=l_{2}=0$; and the Hardy spaces occur at $\alpha=2$ and $\alpha=3$.

The value $\alpha=3$ warrants some special attention. This is the end point of the square-integrable range in the Wallach set, and corresponds to the "limit of holomorphic discrete series" in the sense of Knapp and Okamoto [15]. In particular, we can describe the space $\mathfrak{Y}_{\lambda^{0}, 3}$, for general $\lambda^{0}$, as a Hardy space associated with the rank one boundary component of $H$. Needed for this purpose is another kind of gamma integral. Let $P_{1}$ be the rank-one boundary component of $P$ consisting of all nonnegative $2 \times 2$ matrices $r$ of rank one. In other words, $P_{1}=\left\{r \in \mathrm{C}^{2 \times 2}\right.$ : $r>0, \Delta(r)=0\}$ is the forward light cone. Set

$$
\Gamma^{\lambda^{0}}\left(P_{1}\right)=\int_{P_{1}} e^{-\operatorname{tr}(r)} \lambda^{0}(r, \bar{r}) d m(r)
$$

where $d m(r)$ denotes relatively $A$-invariant measure on $P_{1}$. Explicitly, if we write $r=\left(r_{i j}\right)$ as a $2 \times 2$ matrix, then $d m(r)=r_{11}^{-1} d r_{11} d r_{12}$ and $d m\left(a^{*} r a\right)=|\Delta(a)|^{2} d m(r)$ for $a \in A$. This gamma integral is evaluated in $\$ 8$. The result is as follows. 
THEOREM 6.6. The linear transformation $\Gamma^{\lambda^{0}}\left(P_{1}\right)$ is positive definite. In fact,

$$
\Gamma^{\lambda^{0}}\left(P_{1}\right)=\lim _{\alpha \rightarrow 1}\left\{\Gamma^{\lambda^{0}}(\alpha) / \Gamma(\alpha-1)\right\}=c\left(\lambda^{0}\right) N^{\lambda^{0}}(3)
$$

where $c\left(\lambda^{9}\right)$ is the positive constant $\lim _{\alpha \rightarrow 3}\left\{\psi^{\lambda^{0}}(\alpha-2) /(\alpha-3)\right\}$.

As a corollary, we obtain the description of $\mathfrak{V}_{\lambda^{0}, 3}$ as a Hardy-type Hilbert space. Indeed, let $\mathcal{H}\left(\lambda^{0}, P_{1}\right)$ be the collection of all holomorphic functions $F: H \rightarrow W^{\lambda^{0}}$ such that

$$
\|F\|_{\lambda^{0}, P_{1}}^{2}=\sup _{t \in P}\left\{\int_{S+i P_{1}}\left\|\lambda^{0}(y, \bar{y})^{1 / 2} F(x+i(y+t))\right\|^{2} d x d m(y)\right\}<\infty .
$$

Then $\mathcal{H}\left(\lambda^{0}, P_{1}\right)$ is a Hilbert space (a priori, it could be zero) relative to the inner product derived from the Hardy-type norm (6.9).

Corollary 6.7. The spaces $\mathfrak{Y}_{\lambda^{0}, 3}$ and $\mathcal{H}\left(\lambda^{0}, P^{1}\right)$ coincide. In particular, $\mathcal{H}\left(\lambda^{0}, P^{1}\right)$ $\neq 0$.

This result is due to Rossi and Vergne [17] when $\lambda^{0}=1$ (for general $G$ ), and for general $\lambda^{0}$ to R. A. Kunze and the present authors [8]. The proof of Corollary 6.7 requires a theorem of Paley-Wiener type that is founded upon the Plancherel formula

$$
\begin{aligned}
c\left(\lambda^{0}\right) \int_{P}\left\|\Gamma^{\lambda^{0}}\left(P_{1}\right) \lambda^{0}(r, \bar{r})^{1 / 2} f(r)\right\|^{2} \Delta(r)^{-1} d r \\
\quad=\sup _{t \in P}\left\{\int_{S+i P_{1}}\left\|\lambda^{0}(y, \bar{y})^{1 / 2} F(x+i(y+t))\right\|^{2} d x d m(y)\right\}
\end{aligned}
$$

where $c\left(\lambda^{9}\right)$ is the positive constant $4^{l_{1}+l_{2}-1}$ and $F$ is the Laplace transform (5.5) of $f$. The technical arguments are completely analogous to those in [17].

The value $\alpha=2$ corresponds to a Hardy space associated with the Silov boundary of $H$. However, except for the case $l_{1}=l_{2}=0$, which is the classical Hardy space treated in [17], the Paley-Wiener theorem seems to be more complicated.

We remark that the group $G=U(2,2)$ is a reductive group of real rank two. The results of this section provide the first determination of the complete analytic continuation of the holomorphic discrete series for a group of real rank greater than one. From our results one might conjecture for a group of real rank $m$, that for general $\lambda^{0}$ the analytic continuation will end precisely at $m$ th "integral point" beyond the square-integrable range, although it is known from [17] that when $\lambda^{0}=1$ the analytic continuation goes beyond the $m$ th limit point. For $G=U(2,2)$, this is the content of Theorem 6.5.

Finally, we note that Rossi and Vergne have shown that for $\lambda^{0}=1$ there are discrete points in the Wallach set beyond the analytic continuation. In fact, for $G=U(2,2)$, in the case in which $l_{1}=0$ or $l_{2}=0$ (so that $\Gamma^{\lambda^{0}}$ is scalar-valued), the value $\alpha=1$ is always in the Wallach set. The resulting representations of the 
conformal group arise in the physical applications treated by Jakobsen and Vergne [13]. The explicit realization of such spaces for general $\lambda^{0}$ is an open problem. ${ }^{2}$

7. Miscellaneous properties of the gamma function. Putting aside the applications to harmonic analysis on the conformal group, we can study the matrix-valued gamma function given in Definition 3.2 just as we would any transcendental special function. In fact, the calculations of $\Gamma^{\lambda^{0}}$ in Theorem 4.1 and the trace function $\psi^{\lambda^{0}}$ in Theorem 6.2 allow us to develop for $\Gamma^{\lambda^{0}}$ analogs of some of the well-known properties of the ordinary gamma function. In what follows, we list a few such formulas which are particularly simple. We shall not attempt to compile an exhaustive list.

Fix $\lambda^{0}=\lambda^{0}\left(\cdot ; l_{1}, l_{2}\right) \in \hat{\mathscr{A}}_{0}$. For a given complex number $\alpha$, we adopt the notation

$$
\alpha^{\prime}=\alpha+2 l_{1}+2 l_{2}+2
$$

for the expression on the right of (7.1) arises frequently in the sequel. Note that $\alpha^{\prime}=\alpha+\operatorname{deg}\left(\rho^{l_{1}}\right)+\operatorname{deg}\left(\rho^{l_{2}}\right)$. We begin with a symmetry of the trace function $\psi^{\lambda^{0}}(\alpha)=d_{\lambda}^{-1} \operatorname{tr}\left(\Gamma^{\lambda^{0}}(\alpha)^{-1} \Gamma^{\lambda^{0}}(\alpha+2)\right)$, easily verifiable from Theorem 6.2.

PROPOSITION 7.1. For all complex numbers $\alpha$,

$$
\psi^{\lambda^{0}}(\alpha)=\psi^{\lambda^{0}}\left(2-\alpha^{\prime}\right)
$$

The familiar formula

$$
\Gamma(z) \Gamma(1-z)=\frac{\pi}{\sin \pi z}
$$

for the classical gamma function has the following analog.

PROPOSITION 7.2. For all complex numbers $\alpha$,

$$
\Gamma^{\lambda^{0}}(\alpha) \Gamma^{\lambda^{0}}\left(2-\alpha^{\prime}\right)=\frac{\pi^{2}}{\psi^{\lambda^{0}}(\alpha) \sin \pi \alpha \sin \pi\left(2-\alpha^{\prime}\right)} I
$$

where I denotes the identity transformation.

Finally, there is an analog of Stirling's formula

$$
\Gamma(x)=e^{-x} x^{x-1 / 2}(2 \pi)^{1 / 2}\{1+O(1 / x)\}
$$

which describes the asymptotic behavior of $\Gamma^{\lambda^{0}}$.

Proposition 7.3. As $\alpha \rightarrow \infty, \Gamma^{\lambda^{0}}(\alpha)$ asymptotically approaches a scalar matrix. More precisely,

$$
\Gamma^{\lambda^{0}}(\alpha)=(2 \pi) e^{-2 \alpha} \alpha^{-4+\alpha+\alpha^{\prime}}(I+O(1 / \alpha))
$$

where $I$ is the identity transformation on $W^{\lambda^{0}}$.

We could proceed further with analogs of the Euler product formula, the infinite series for the logarithmic derivative, and various other properties of the classical

\footnotetext{
${ }^{2}$ Added in proof. It is shown by F. Williams (University of Massachusetts) in a recent preprint Unitarizable highest weight modules of the conformal group that for $l_{1} \neq 0$ and $l_{2} \neq 0$ there are no such discrete points in the Wallach set. Thus, for general $\lambda^{0}$ the Wallach set is precisely the closed interval $[2, \infty)$.
} 
gamma function. These formulas, however, are somewhat more messy. In addition, there are curious symmetries that relate to the dependence of the generalized gamma function on both $\lambda^{0}$ and $\alpha$ (cf., Proposition 8.1).

8. Proofs of the main theorems and formulas for the matrix entries. We start with Theorem 4.1 and calculate the eigenvalues (4.2) of the gamma function. The technique involves the detailed fine structure from Appendix B, and we freely use the notation given there. However, as indicated in $\$ 4$ the idea underlying the calculation is the commutation relation (3.12). Indeed, $\Gamma^{\lambda^{0}}(\alpha)$ commutes with the representation $u \rightarrow \lambda^{0}(u, \bar{u})$ of $U=U(2)$; or equivalently, the tensor product representation $a \rightarrow \lambda^{0}\left(a, a^{\vee}\right)$ of $A=G L(2, \mathrm{C})$, where $a^{\vee}=a^{t^{-1}}$. Observe from (3.2) and the identity $a^{\vee}=\Delta(a)^{-1}$ pap ${ }^{-1}$, where $p=\left(\begin{array}{rr}0 & 1 \\ -1 & 0\end{array}\right)$, that

$$
\lambda^{0}\left(a, a^{\vee}\right)=\Delta(a)^{-2 l_{2}} \rho^{l_{1}}(a) \otimes\left(T \rho^{l_{2}}(a) T^{-1}\right)
$$

where $T=\rho^{l_{2}}(p)$. In particular, the representation $a \rightarrow \lambda^{0}\left(a, a^{\vee}\right)$ is equivalent to the representation $\Delta^{-2 l_{2}} \rho^{l_{1}} \otimes \rho^{l_{2}}$ of $A$, so $\Gamma^{\lambda^{0}}(\alpha)$ is diagonalized by a variant (determined by $T$; viz. (8.15)) of the Clebsch-Gordan transformation (cf. (B.5) and (B.6)) for the space $W^{l_{1}, l_{2}}=V^{l_{1}} \otimes V^{l_{2}}$.

We shall perform the integration in (3.11). As in Appendix B, let $e_{m_{1}}^{l_{1}} \otimes e_{m_{2}}^{l_{2}}$ and $E_{l m}^{l_{1}, l_{2}}$ be the Gelfand-Tsetlin and Clebsch-Gordan bases, respectively, of $W^{l_{1}, l_{2}}=$ $W^{\lambda^{0}}$. Note that $\bar{r}=r^{t}$ and $\rho^{l_{2}}\left(r^{t}\right)=\rho^{l_{2}}(r)^{t}$ for $r$ in $P$. Thus, in the former basis

$$
\lambda^{0}\left(r, r^{t}\right)_{m_{1}^{\prime} m_{2}^{\prime}, m_{1} m_{2}}=D_{m_{1}^{\prime} m_{1}}^{l_{1}}(r) D_{m_{2} m_{2}^{\prime}}^{l_{2}^{\prime}}(r)
$$

or by (B.14)

$$
\begin{aligned}
\lambda^{0}\left(r, r^{t}\right)_{m_{1}^{\prime} m_{2}^{\prime}, m_{1}, m_{2}}= & \sum_{l=\left|l_{1}-l_{2}\right|}^{l_{1}+l_{2}} C_{m_{1}^{\prime}, m_{2}, m_{1}^{\prime}+m_{2}}^{l_{1}, l_{2}, l} \Delta(r)^{l_{1}+l_{2}-l} \\
& \times D_{m_{1}^{\prime}+m_{2}, m_{1}+m_{2}^{\prime}}^{l}(r) C_{m_{1}, m_{2}^{\prime}, m_{1}+m_{2}^{\prime}}^{l_{1}, l_{2}, l}
\end{aligned}
$$

We next introduce "polar coordinates" [12; §3.3] into the integration in (3.11). Thus, let $r=u a u^{*}$ with $u \in U$ and $a=\operatorname{diag}\left(a_{1}, a_{2}\right)$ with $a_{1}>0$ and $a_{2}>0$. Then

$$
d_{*} r=\frac{d r}{\Delta(r)^{2}}=\frac{\left(a_{1}-a_{2}\right)^{2} d a_{1} d a_{2} d u}{2\left(a_{1} a_{2}\right)^{2}}
$$

where $d a_{1}$ and $d a_{2}$ denote Lebesgue measure on the positive reals and $d \mu$ is normalized Haar measure on $U$.

Note from (B.3) that

$$
D_{m m}^{l}(a)=\left(a_{1}\right)^{l+m}\left(a_{2}\right)^{l-m}
$$

for $a=\operatorname{diag}\left(a_{1}, a_{2}\right)$, and the off-diagonal entries of $D^{\prime}(a)$ vanish. Upon substitution of (8.3) and (8.4) into (3.11), we obtain

$$
\begin{aligned}
\Gamma^{\lambda^{0}}(\alpha)_{m_{1}^{\prime} m_{2}^{\prime}, m_{1} m_{2}}= & \frac{1}{2} \int_{0}^{\infty} \int_{0}^{\infty} \sum_{l, m}\left(a_{1} a_{2}\right)^{\alpha+l_{1}+l_{2}-l-2}\left(a_{1}-a_{2}\right)^{2} e^{-a_{1}-a_{2}} \\
& \times C_{m_{1}^{\prime}, m_{2}, m_{1}^{\prime}+m_{2}}^{l_{1} l_{l}, l} \int_{U} D_{m_{1}^{\prime}+m_{2}, m}^{l}(u) D_{m m}^{l}(a) D_{m, m_{1}+m_{2}^{\prime}}^{l}\left(u^{*}\right) d u \\
& \times C_{m_{1}, m_{2}, m_{1}+m_{2}^{\prime}}^{l_{1}, l_{2}, l} d a_{1} d a_{2} .
\end{aligned}
$$


From Schur orthogonality, the integral over $U$ is evaluated as

$$
\int_{U} D_{m_{1}^{\prime}+m_{2}, m}^{l}(u) D_{m, m_{1}+m_{2}^{\prime}}^{l}\left(u^{*}\right) d u=(2 l+1)^{-1} \delta_{m_{1}^{\prime}+m_{2}, m_{1}+m_{2}^{\prime}}
$$

and from (8.5) and the formula

$$
\sum_{m=-l}^{l}\left(a_{1}\right)^{l+m}\left(a_{2}\right)^{l-m}=\frac{\left(a_{1}\right)^{2 l+1}-\left(a_{2}\right)^{2 l+1}}{a_{1}-a_{2}}
$$

the sum over $m$ is evaluated. Then (8.6) becomes

$$
\begin{aligned}
\Gamma^{\lambda^{0}}(\alpha)_{m_{1}^{\prime} m_{2}^{\prime}, m_{1} m_{2}}= & \frac{1}{2} \sum_{l} \int_{0}^{\infty} \int_{0}^{\infty}\left(a_{1} a_{2}\right)^{\alpha+l_{1}+l_{2}-l-2}\left(a_{1}-a_{2}\right)\left(a_{1}^{2 l+1}-a_{2}^{2 l+1}\right) e^{-a_{1}-a_{2}} \\
& \times(2 l+1)^{-1} C_{m_{1}^{\prime}, m_{2}, m_{1}^{\prime}+m_{2}}^{l_{1}, l_{2}, l} C_{m_{1}, m_{2}, m_{1}+m_{2}^{\prime} \delta_{m_{1}^{\prime}+m_{2}, m_{1}+m_{2}^{\prime}}^{l_{1}, l_{2}, l} d a_{1} d a_{2}}
\end{aligned}
$$

or

$$
\begin{aligned}
\Gamma^{\lambda^{0}}(\alpha)_{m_{1}^{\prime} m_{2}^{\prime}, m_{1} m_{2}}= & \sum_{l} \Gamma\left(\alpha+l_{1}+l_{2}-l-1\right) \\
& \times \Gamma\left(\alpha+l_{1}+l_{2}+l\right) C_{m_{1}^{\prime}, m_{2}, m_{1}^{\prime}+m_{2}}^{l_{1}, l_{2},} C_{m_{1}, m_{2}^{\prime}, m_{1}+m_{2}^{\prime}}^{l_{1}, l_{m_{1}}, l m_{2}, m_{1}+m_{2}^{\prime}}
\end{aligned}
$$

Since the row indices $m_{1}^{\prime} m_{2}^{\prime}$ and column indices $m_{1} m_{2}$ appear in different Wigner coefficients, it is convenient to apply the symmetries (B.11) to put the right side of (8.10) in the form

$$
\begin{aligned}
\sum_{l} \Gamma(\alpha+ & \left.l_{1}+l_{2}-l-1\right) \Gamma\left(\alpha+l_{1}+l_{2}+l\right) \\
& \times(-1)^{l_{2}+m_{2}^{\prime}}(-1)^{2 l_{2}}\left\{(2 l+1) /\left(2 l_{1}+1\right)\right\}^{1 / 2} \\
& \times C_{m_{1}^{\prime}, m_{2}, m_{1}^{\prime}+m_{2}}^{l_{1}, l_{2}, l} C_{m_{1}+m_{2}^{\prime},-m_{2}^{\prime}, m_{1}}^{l, l_{2}, l_{1}} \delta_{m_{1}^{\prime}+m_{2}, m_{1}+m_{2}^{\prime}}
\end{aligned}
$$

Upon substitution of (B.20), the expression (8.11) becomes

$$
\begin{aligned}
\sum_{l, L} \Gamma(\alpha+ & \left.l_{1}+l_{2}-l-1\right) \Gamma\left(\alpha+l_{1}+l_{2}+l\right) \\
& \times(-1)^{l_{2}+m^{\prime}}(-1)^{2 l_{1}+2 l_{2}}(2 l+1)\left\{(2 L+1) /\left(2 l_{1}+1\right)\right\}^{1 / 2} \\
& \times\left\{\begin{array}{c}
l_{2} l_{1} L \\
l_{2} l_{1} l
\end{array}\right\} C_{-m_{2}^{\prime}, m_{1}^{\prime}, m_{1}^{\prime}-m_{2}^{\prime}}^{l_{2} l_{1}, L} C_{m_{1}-m_{2}, m_{2}, m_{1}}^{L, l_{2}, l_{m_{1}^{\prime}-m_{2}^{\prime}, m_{1}-m_{2}},}
\end{aligned}
$$

or equivalently, by (B.11)

$$
\begin{aligned}
\sum_{l, L} \Gamma\left(\alpha+l_{1}+l_{2}-l-1\right) \Gamma(\alpha & \left.+l_{1}+l_{2}+l\right)(-1)^{l_{2}+m_{2}^{\prime}}(-1)^{l_{2}+m_{2}}(-1)^{2 l_{2}+2 l_{1}}(2 l+1) \\
& \times\left\{\begin{array}{c}
l_{2} l_{1} L \\
l_{2} l_{1} l
\end{array}\right\} C_{-m_{2}^{\prime}, m_{1}^{\prime}, m_{1}^{\prime}-m_{2}^{\prime}}^{l_{2}, L} C_{-m_{2}, m_{1}, m_{1}-m_{2}}^{l_{2}, L} \delta_{m_{1}^{\prime}-m_{2}^{\prime}, m_{3}-m_{2} .}
\end{aligned}
$$

Here, we have used the fact that $(-1)^{2\left(l_{1}+l_{2}-L\right)}=1$ and $\delta_{m_{1}^{\prime}+m_{2}, m_{1}+m_{2}^{\prime}}=$ $\delta_{m_{1}^{\prime}-m_{2}^{\prime}, m_{1}-m_{2}}$ 
In the form (8.13), we can see that the Clebsch-Gordan basis diagonalizes the gamma function. Indeed, we can rewrite (8.13) as

$$
\Gamma^{\lambda^{0}}(\alpha)_{m_{1}^{\prime} m_{2}^{\prime}, m_{1} m_{2}}=\sum_{L=\left|l_{1}-l_{2}\right|}^{l_{1}+l_{2}} B_{m_{1}^{\prime}, m_{2}^{\prime}, M^{\prime}}^{l_{1}, l_{2}, L^{\prime}} \Gamma^{\lambda^{0}}(\alpha)_{L^{\prime} M^{\prime}, L M} B_{m_{1}, m_{2}, M^{\prime}}^{l_{1}, l_{2}, L} \delta_{M^{\prime} M^{\prime}} \delta_{L^{\prime} L}
$$

where the $B$-coefficients are given in terms of the Wigner coefficients as

$$
B_{m_{1}, m_{2}, m}^{l_{1}, l_{2}, l}=(-1)^{l_{2}+m_{2}} C_{-m_{2}, m_{1}, m}^{l_{2}, l_{1}, l}
$$

and

$$
\begin{aligned}
\Gamma^{\lambda^{0}}(\alpha)_{L^{\prime} M^{\prime}, L M}= & \delta_{L^{\prime} L} \delta_{M^{\prime} M} \sum_{l=\left|l_{1}-l_{2}\right|}^{l_{1}+l_{2}} \Gamma\left(\alpha+l_{1}+l_{2}-l-1\right) \\
& \times \Gamma\left(\alpha+l_{1}+l_{2}+l\right)(2 l+1)(-1)^{2 l_{1}+2 l_{2}}\left\{\begin{array}{c}
l_{2} l_{1} L \\
l_{2} l_{1} l
\end{array}\right\} .
\end{aligned}
$$

Thus, in the notation of Theorem 4.1, $\Gamma^{\lambda^{0}}(\alpha)$ can be diagonalized, the eigenvalues are

$$
\gamma_{L}^{\lambda^{0}}(\alpha)=\sum_{l} \Gamma\left(\alpha+l_{1}+l_{2}-l-1\right) \Gamma\left(\alpha+l_{1}+l_{2}+l\right)(2 l+1)(-1)^{2 l_{1}+2 l_{2}}\left\{\begin{array}{c}
l_{2} l_{1} L \\
l_{2} l_{1} l
\end{array}\right\}
$$

for $\left|l_{1}-l_{2}\right| \leqslant L<l_{1}+l_{2}$, and the $L$ th eigenvalue appears with multiplicity $(2 L+$ 1). It remains to be shown that (8.17) can be put in the form (4.2). We should note that the matrices $\Gamma^{\lambda^{0}}(\alpha)$ and $\tilde{\Gamma}^{\tilde{\lambda}^{0}}(\alpha)$ commute with one another and are both diagonalized by transformation by the matrix (8.15). On the other hand, $\tilde{\Gamma}^{\lambda^{0}}(\alpha)$ is diagonalized by the Wigner coefficient $C_{m_{2} m_{1} m}^{l_{2} l_{1} l}$.

If we substitute the expression (B.21) for the $6-j$ symbol into (8.17), we obtain a double sum over $l$ and $n$. The sum over $l$ is

$$
\begin{aligned}
& \sum_{l=\left|l_{1}-l_{2}\right|}^{l_{1}+l_{2}}(2 l+1) \Gamma\left(\alpha+l_{1}+l_{2}-l-1\right) \Gamma\left(\alpha+l_{1}+l_{2}+l\right) \\
& \quad \times \frac{\left(l+l_{1}-l_{2}\right) !\left(l+2 l_{2}-L-n\right) !}{\left(l+l_{2}-l_{1}\right) !\left(l_{1}+l_{2}+l+1\right) !\left(l_{1}+l_{2}-l\right) !\left(l-2 l_{2}+L+n\right) !}
\end{aligned}
$$

which by a change of variable (say $x=l+l_{2}-l_{1}$ ) takes the form

$$
\left.\begin{array}{l}
\frac{\left(2 l_{1}-2 l_{2}+1\right) ! \Gamma\left(\alpha+2 l_{2}-1\right) \Gamma\left(\alpha+2 l_{1}\right)\left(l_{1}+l_{2}-L-n\right) !}{\left(2 l_{1}+1\right) !\left(2 l_{2}\right) !\left(l_{1}-3 l_{2}+L+n\right) !} \\
\quad \times_{5} F_{4}\left(\begin{array}{ll}
2 l_{1}-2 l_{2}+1, & l_{1}-l_{2}+\frac{3}{2}, \alpha+2 l_{1},-2 l_{2}, l_{1}+l_{2}-L-n+1 \\
l_{1}-l_{2}+\frac{1}{2},-\alpha-2 l_{2}+2,2 l_{1}+2, l_{1}-3 l_{2}+L+n+1
\end{array} \mid 1\right.
\end{array}\right) .
$$

Here, we use the notation of Appendix A. Note that the gamma matrix is symmetric in $l_{1}$ and $l_{2}$, so we lose no generality in assuming that $l_{1}<l_{2}$. Since the hypergeometric series above is well poised, by formula (A.4) the expression (8.19) is simply

$$
\frac{\left(l_{1}+l_{2}-L-n\right) ! \Gamma\left(\alpha+2 l_{1}\right) \Gamma(\alpha-1) \Gamma\left(\alpha+3 l_{2}+l_{1}-L-n\right)}{\left(2 l_{2}\right) !\left(l_{1}-l_{2}+L+n\right) ! \Gamma\left(\alpha+l_{1}+l_{2}-L-n\right)} .
$$


To finish the calculation, substitute (B.21) and (8.20) into (8.17). Then

$$
\begin{aligned}
\gamma_{L}^{\lambda^{0}}(\alpha)= & \frac{\Gamma\left(\alpha+2 l_{1}\right) \Gamma(\alpha-1)\left(L+l_{2}-l_{1}\right) !\left(l_{1}+l_{2}+L+1\right) !\left(l_{1}+l_{2}-L\right) !}{\left(L+l_{1}-l_{2}\right) !\left(2 l_{2}\right) !} \\
& \times \sum_{n} \frac{(-1)^{n}}{n !} \frac{\left(L+l_{1}-l_{2}+n\right) ! \Gamma\left(\alpha+3 l_{2}-l_{1}-L-n\right)}{\left(l_{1}+l_{2}-L-n\right) !(2 L+1+n) ! \Gamma\left(\alpha+l_{1}+l_{2}-L-n\right)} \\
= & \frac{\left(L+l_{2}-l_{1}\right) !\left(l_{1}+l_{2}+L+1\right) ! \Gamma\left(\alpha+2 l_{1}\right) \Gamma(\alpha-1) \Gamma\left(\alpha+3 l_{2}+l_{1}-L\right)}{\left(2 l_{2}\right) !(2 L+1) ! \Gamma\left(\alpha+l_{1}+l_{2}-L\right)} \\
& \times{ }_{3} F_{2}\left(\begin{array}{r}
L-l_{1}-l_{2}+1,-l_{1}-l_{2}+L,-\alpha-l_{1}-l_{2}+L+1 \\
2 L+2,-\alpha-3 l_{2}-l_{1}+L+1
\end{array}\right) .
\end{aligned}
$$

Finally, the summation theorem (A.3) applies, and (8.21) becomes (4.2),

$$
\gamma_{L}^{\lambda^{0}}(\alpha)=\frac{\Gamma\left(\alpha+2 l_{1}\right) \Gamma\left(\alpha+2 l_{2}\right) \Gamma\left(\alpha+2 l_{1}+2 l_{2}+1\right) \Gamma(\alpha-1)}{\Gamma\left(\alpha+l_{1}+l_{2}-L\right) \Gamma\left(\alpha+l_{1}+l_{2}+L+1\right)}
$$

This completes the proof of Theorem 4.1.

We can now verify formula (4.7) for the eigenvalues of $\tilde{\Gamma}^{\lambda^{0}}(\alpha)$. Indeed, (4.7) follows from (4.6) when the matrix $\lambda^{0}(r, r)$ is transformed to the Clebsch-Gordan basis and the integrations are performed in analogy to the process described here for the evaluation of (8.6).

We next prove Theorem 6.2 by calculating formula (6.3) for the trace function. By (8.22),

$$
\begin{aligned}
d_{\lambda} \psi^{\lambda^{0}}(\alpha-2)= & \frac{\Gamma\left(\alpha+2 l_{1}\right) \Gamma\left(\alpha+2 l_{2}\right) \Gamma(\alpha-1) \Gamma\left(\alpha+2 l_{1}+2 l_{2}+1\right)}{\Gamma\left(\alpha+2 l_{1}-2\right) \Gamma\left(\alpha+2 l_{2}-2\right) \Gamma(\alpha-3) \Gamma\left(\alpha+2 l_{1}+2 l_{2}-1\right)} \\
& \times \sum_{L=\left|l_{1}-l_{2}\right|}^{l_{1}+l_{2}}(2 L+1) \frac{\Gamma\left(\alpha+l_{1}+l_{2}-L-2\right) \Gamma\left(\alpha+l_{1}+l_{2}+L-1\right)}{\Gamma\left(\alpha+l_{1}+l_{2}-L\right) \Gamma\left(\alpha+l_{1}+l_{2}+L+1\right)}
\end{aligned}
$$

where we have used the fact that the $L$ th eigenvalue of $\Gamma^{\lambda^{0}}(\alpha)$ has multiplicity $(2 L+1)$. The problem is to evaluate the sum over $L$.

Consider the more general problem

$$
\sum_{L=\left|l_{1}-l_{2}\right|}^{l_{1}+l_{2}}(2 L+1) \frac{\Gamma\left(\alpha+l_{1}+l_{2}-L-n\right) \Gamma\left(\alpha+l_{1}+l_{2}-L-n+1\right)}{\Gamma\left(\alpha+l_{1}+l_{2}-L\right) \Gamma\left(\alpha+l_{1}+l_{2}+L+1\right)}
$$

for positive integer $n$. Ultimately, we will set $n=2$. The trick here is to insert gamma functions in the denominator which automatically prescribe the limits of summation by containing negative integer arguments outside of those limits. Thus, we multiply by the factors

$$
\frac{\Gamma\left(l_{1}+l_{2}+L+x+1\right)}{\left(l_{1}+l_{2}-L\right) !} \text { and } \frac{\Gamma\left(L+l_{1}-l_{2}-x+1\right)}{\left(L+l_{1}-l_{2}\right) !}
$$

to set the limits of summation (here, we make the choice $l_{1}<l_{2}$ which does not impair the generality of the result), as well as the factors

$$
\frac{\Gamma\left(l_{1}+l_{2}+L+x+2\right)}{\left(l_{1}+l_{2}+L+1\right) !} \text { and } \frac{\left(L-l_{1}+l_{2}\right) !}{\Gamma\left(L-l_{1}+l_{2}+x+1\right)}
$$


which keep the hypergeometric series well poised. Then (8.24) is rewritten as

$$
\begin{aligned}
\lim _{x \rightarrow 0} \sum_{L}(2 L+1) & \frac{\Gamma\left(\alpha+l_{1}+l_{2}-L-n\right) \Gamma\left(\alpha+l_{1}+l_{2}+L-n+1\right) \Gamma\left(l_{1}+l_{2}-L+x+1\right)}{\Gamma\left(\alpha+l_{1}+l_{2}-L\right) \Gamma\left(\alpha+l_{1}+l_{2}+L+1\right) \Gamma\left(L-l_{1}+l_{2}+x+1\right)} \\
& \times \frac{\Gamma\left(l_{1}+l_{2}+L+x+2\right) \Gamma\left(L+l_{1}-l_{2}-x+1\right)\left(L-l_{1}+l_{2}\right) !}{\left(l_{1}+l_{2}-L\right) !\left(l_{1}+l_{2}+L+1\right) !\left(L+l_{1}-l_{2}\right) !}
\end{aligned}
$$

To evaluate this sum, we make a change of variables, say $l=L+l_{1}-l_{2}$. Then (8.24) is expressed in terms of well-poised ${ }_{7} F_{6}$ as

$$
\begin{aligned}
\lim _{x \rightarrow 0} & {\left[\frac{\left(2 l_{2}-2 l_{1}+1\right) ! \Gamma\left(\alpha+2 l_{1}-n\right) \Gamma\left(\alpha+2 l_{2}-n+1\right) \Gamma\left(2 l_{1}+x+1\right) \Gamma\left(2 l_{2}+x+2\right) \Gamma(1-x)}{\left(2 l_{1}\right) !\left(2 l_{2}+1\right) ! \Gamma\left(\alpha+2 l_{1}\right) \Gamma\left(\alpha+2 l_{2}+1\right) \Gamma\left(2 l_{2}-2 l_{1}+x+1\right)}\right.} \\
& \left.\times{ }_{7} F_{6}\left(\begin{array}{rr}
2 l_{2}-2 l_{1}+1, l_{2}-l_{1}+\frac{3}{2}, 1-x, & -\alpha-2 l_{1}+1, \alpha+2 l_{2}-n+1,2 l_{2}+x+2,-2 l_{1} \\
l_{2}-l_{1}+\frac{1}{2}, 2 l_{2}-2 l_{1}+x+1, & \alpha+2 l_{2}+1,-\alpha-2 l_{1}+n+1,-2 l_{1}-x, 2 l_{2}+2
\end{array}\right)\right] .
\end{aligned}
$$

Notice that only in the case $n=2$ is Dougall's theorem (A.5) applicable, and that is the case of interest to us. Thus, take $n=2$; substitute from (A.5) into (8.28); take the limit as $x \rightarrow 0$; substitute the resulting expression for the sum in (8.23); and finally, replace $\alpha$ by $\alpha+2$. The result is the desired formula (6.3).

At this point it is appropriate to list some formulas for the matrix entries of the gamma function.

Proposition 8.1. Relative to the Gelfand-Tsetlin basis, the gamma function has matrix entries $\Gamma^{\lambda}(\alpha)_{m_{1}^{\prime} m_{2}^{\prime}, m_{1} m_{2}}$ given by formula (8.10), and the corresponding result for the inverse gamma function is

$$
\begin{aligned}
& \left(\Gamma^{\lambda^{0}}(\alpha)^{-1}\right)_{m_{1}^{\prime} m_{2}^{\prime} ; m_{1} m_{2}} \\
& =\psi^{\lambda^{0}}(\alpha) \sum_{l=\left|l_{1}-l_{2}\right|}^{l_{1}+l_{2}} \frac{C_{m_{1}^{\prime}, m_{2}, m_{1}^{\prime}+m_{2}}^{l_{1} l_{2}, l} C_{m_{1}, m_{2}^{\prime}, m_{1}+m_{2}^{\prime}}^{l_{1}, l_{2}, l}}{\Gamma\left(\alpha+l_{1}+l_{2}-l+1\right) \Gamma\left(\alpha+l_{1}+l_{2}+l+2\right)} .
\end{aligned}
$$

Moreover, there is a curious symmetry

$$
\Gamma^{\lambda^{0}}(\alpha)_{m_{1}^{\prime} m_{2}^{\prime}, m_{1} m_{2}}=\Gamma^{\mu^{0}}(\alpha)_{n_{1}^{\prime} n_{2}^{\prime}, n_{1} n_{2}}
$$

where $\lambda^{0}=\lambda^{0}\left(\cdot ; l_{1}, l_{2}\right), \mu^{0}=\lambda^{0}\left(\cdot ; \frac{1}{2}\left(l_{1}+l_{2}+m_{1}^{\prime}+m_{2}\right), \frac{1}{2}\left(l_{1}+l_{2}-m_{1}^{\prime}-m_{2}\right)\right)$, and $n_{1}^{\prime}=\frac{1}{2}\left(l_{1}-l_{2}+m_{1}^{\prime}-m_{2}\right), n_{2}^{\prime}=\frac{1}{2}\left(l_{1}-l_{2}-m_{1}-m_{2}^{\prime}\right), n_{1}=\frac{1}{2}\left(l_{1}-l_{2}+m_{1}-\right.$ $\left.m_{2}^{\prime}\right), n_{2}=\frac{1}{2}\left(l_{1}-l_{2}-m_{1}^{\prime}+m_{2}\right)$.

Since we know that the matrix entries $\Gamma^{\lambda^{0}}(\alpha)^{-1}$ in the Clebsch-Gordan basis are just the eigenvalues $\gamma_{l}^{\lambda^{0}}(\alpha)^{-1}$, formula (8.29) is derived in analogy to the preceding calculation. (8.30) follows from (8.10) by an application of the last symmetry, the so-called Regge transposition, in (B.11). We omit the details.

Finally, we calculate the gamma function $\Gamma^{\lambda^{0}}\left(P_{1}\right)$ for the boundary $P_{1}$ of $P$, given by (6.8).

Recall that $P_{1}$ consists of all nonnegative $2 \times 2$ matrices $r$ such that $\Delta(r)=0$. The group $A=G L(2, \mathrm{C})$ acts transitively on $P_{1}$, and $P_{1}$ is equivalent as a right 
$A$-space to $Z \backslash A$ where $Z$ is the stability group of the point $1=\left(\begin{array}{ll}1 & 9 \\ 0 & 0\end{array}\right)$. Clearly,

$$
z=\left(\begin{array}{ll}
u & 0 \\
\gamma & \delta
\end{array}\right), \quad|u|=1 \text { and } \gamma, \delta \in \mathbf{C}, \delta \neq 0 .
$$

Let $T$ be the subgroup of $A$ of the form

$$
t=\left(\begin{array}{ll}
\alpha & \beta \\
0 & 1
\end{array}\right), \quad \alpha>0, \beta \in \mathbf{C} .
$$

Then the set $Z T$ is open and dense in $A$, and the mapping $t \rightarrow r=t^{*} 1 t$ identifies $T$ with $P_{1}$. Specifically,

$$
r=t^{* 1} t=\left(\begin{array}{cc}
\alpha^{2} & \alpha \bar{\beta} \\
\alpha \beta & |\beta|^{2}
\end{array}\right)
$$

in " $t$-coordinates", and

$$
r=\left(\begin{array}{cc}
x_{1} & \bar{z} \\
z & x_{1}^{-1}|z|^{2}
\end{array}\right), \quad z=x_{2}+i x_{3}, x_{1}>0
$$

in "Euclidean coordinates". The measure

$$
d m(r)=c x_{1}^{-1} d x_{1} d x_{2} d x_{3}
$$

is relatively $A$-invariant, i.e.,

$$
d m\left(a^{*} r a\right)=c \Delta(a)^{2} d m(r), \quad a \in A .
$$

Set the positive constant $c$ equal to $\pi$. Then in $t$-coordinates,

$$
d m(t)=\frac{2}{\pi} \alpha d \alpha d \beta
$$

Thus,

$$
\begin{aligned}
\Gamma^{\lambda^{0}}\left(P_{1}\right) & =\int_{P_{1}} e^{-\operatorname{tr} r} \lambda^{0}(r, \bar{r}) d m\left(r^{t}\right) \\
& =\frac{2}{\pi} \int_{0}^{\infty} \int_{\mathrm{C}} e^{-\alpha^{2}-|\beta|^{2} \lambda^{0}\left(t^{*} 1 t, \overline{t^{*} 1 t}\right) \alpha d \alpha d \beta}
\end{aligned}
$$

We shall calculate its eigenvalues and see that $\Gamma^{\lambda^{0}}\left(P_{1}\right)$ is positive-definite.

Since $\bar{r}=r^{t}$ and $\Delta(r)=0$, by (B.14)

$$
\begin{aligned}
\lambda^{0}\left(r, r^{t}\right)_{m_{1}^{\prime} m_{2}^{\prime}, m_{1} m_{2}} & =D_{m_{1}^{\prime} m_{2}}^{l_{1}}(r) D_{m_{2}^{\prime} m_{2}}^{l_{2}}\left(r^{t}\right) \\
& =C_{m_{1}^{\prime}, m_{2}, m_{1}^{\prime}+m_{2}}^{l_{1} l_{2}, l_{1}+l_{2}} C_{m_{1}, m_{2}^{\prime}, m_{1}+m_{2}^{\prime}}^{l_{1}, l_{2}, l_{1}+l_{2}} D_{m_{1}^{\prime}+m_{2}, m_{1}+m_{2}^{\prime}}^{l_{1}+l_{2}}(r) .
\end{aligned}
$$

By (B.3), (A.2), and (8.33)

$$
\begin{aligned}
& D_{m_{1}+m_{2}, m_{1}+m_{2}^{\prime}}^{l_{1}+l_{2}}(r) \\
& =\frac{\left(2 l_{1}+2 l_{2}\right) !\left(\alpha^{2}\right)^{m_{1}^{\prime}+m_{2}^{\prime}+m_{1}+m_{2}}(\alpha \bar{\beta})^{l_{1}+l_{2}-m_{1}-m_{2}^{\prime}}(\alpha \beta)^{l_{1}+l_{2}-m_{1}^{\prime}-m_{2}}}{\left[\left(l_{1}+l_{2}-m_{1}^{\prime}-m_{2}\right) !\left(l_{1}+l_{2}+m_{1}^{\prime}+m_{2}\right) !\left(l_{1}+l_{2}-m_{1}-m_{2}^{\prime}\right) !\left(l_{1}+l_{2}+m_{1}+m_{2}^{\prime}\right) !\right]^{1 / 2}} .
\end{aligned}
$$


Thus, in polar coordinates $\beta=\rho^{e^{i \phi}}, d \beta=\rho d \rho d \phi$, and (8.38) becomes

$$
\begin{aligned}
& \Gamma^{\lambda^{0}}\left(P_{1}\right)_{m_{1}^{\prime} m_{2}^{\prime}, m_{1} m_{2}}=\frac{1}{2 \pi} \int_{0}^{\infty} \int_{0}^{\infty} e^{-\alpha^{2}-\rho^{2}}\left(\alpha^{2}\right)^{l_{1}+l_{2}+\left(m_{1}^{\prime}+m_{2}^{\prime}+m_{1}+m_{2}\right) / 2}\left(\rho^{2}\right)^{l_{1}+l_{2}-\left(m_{1}+m_{2}+m_{1}+m_{2}\right) / 2} \\
& \times C_{m_{1}, m_{2}, m_{1}^{1}+m_{2}}^{l_{1}, l_{2}, l_{1}+l_{2}} C_{m_{1}, m_{2}, m_{1}+m_{2}^{\prime}}^{l_{1}, l_{2}, l_{1}+l_{2}} \frac{\left(2 l_{1}+2 l_{2}\right) !}{\left(l_{1}+l_{2}+m_{1}^{\prime}+m_{2}\right) !\left(l_{1}+l_{2}-m_{1}^{\prime}-m_{2}\right) !} \\
& \times \frac{1}{\left(l_{1}+l_{2}+m_{1}+m_{2}^{\prime}\right) !\left(l_{1}+l_{2}-m_{1}-m_{2}^{\prime}\right) !} \\
& \times\left(\int_{0}^{2 \pi} e^{i\left(m_{1}+m_{2}^{\prime}-m_{1}^{\prime}-m_{2}\right) \phi} d \phi\right) d\left(\rho^{2}\right) d\left(\alpha^{2}\right)
\end{aligned}
$$

which simplifies to

$$
\Gamma^{\lambda^{0}}\left(P_{1}\right)_{m_{1}^{\prime} m_{2}^{\prime}, m_{1} m_{2}}=C_{m_{1}^{\prime}, m_{2}, m_{1}^{\prime}+m_{2}}^{l_{1}, l_{2}, l_{1}+l_{2}} C_{m_{1}, m_{2}^{\prime}, m_{1}+m_{2}^{\prime}}^{l_{1}, l_{2}, l_{1}+l_{2}}\left(2 l_{1}+2 l_{2}\right) ! \delta_{m_{1}^{\prime}-m_{2}^{\prime}, m_{1}-m_{2}} .
$$

In complete analogy to our calculation of (8.17), we use the symmetries (B.11) and the relation (B.21) to put (8.42) in the form

$$
\Gamma^{\lambda^{0}}\left(P_{1}\right)_{m_{1}^{\prime} m_{2}^{\prime}, m_{1} m_{2}}=\sum_{L=\left|l_{1}-l_{2}\right|}^{l_{1}+l_{2}} B_{m_{1}^{\prime}, m_{2}^{\prime}, M^{\prime}}^{l_{1}, l_{2}, \Gamma^{\prime}} \Gamma^{\lambda^{0}}\left(P_{1}\right)_{L^{\prime} M^{\prime}, L M^{\prime}} B_{m_{1}, m_{2}, M}^{l_{1}, l_{2}, L}
$$

where the $B$-coefficients are given by $(8.15)$ and

$$
\begin{aligned}
\Gamma^{\lambda^{0}}\left(P_{1}\right)_{L^{\prime} M^{\prime}, L M} & =\delta_{L^{\prime} L} \delta_{M^{\prime} M}\left(2 l_{1}+2 l_{2}+1\right) !(-1)^{2 l_{1}+2 l_{2}}\left\{\begin{array}{lll}
l_{2} & l_{1} & L \\
l_{2} & l_{1} & l_{1}+l_{2}
\end{array}\right\} \\
& =\delta_{L^{\prime} L} \delta_{M^{\prime} M} \frac{\left(2 l_{1}\right) !\left(2 l_{2}\right) !\left(2 l_{1}+2 l_{2}+1\right) !}{\left(l_{1}+l_{2}-L\right) !\left(l_{1}+l_{2}+L+1\right) !}
\end{aligned}
$$

Consequently, $\Gamma^{\lambda^{0}}\left(P_{1}\right)$ can be diagonalized, and the eigenvalues are the positive numbers

$$
\gamma_{L}^{\lambda^{0}}\left(P_{1}\right)=\frac{\left(2 l_{1}\right) !\left(2 l_{2}\right) !\left(2 l_{1}+2 l_{2}+1\right) !}{\left(l_{1}+l_{2}-L\right) !\left(l_{1}+l_{2}+L+1\right) !} .
$$

This completes the proof of Theorem 6.6.

9. Concluding remarks. Our knowledge of the gamma function associated to the conformal group leads to analogs for the matrix space $\mathbf{C}^{2 \times 2}$ of many classical results of importance in number theory and mathematical physics.

For example, the usual binomial theorem $(1+z)^{n}=\sum_{L=0}^{n}\left(\begin{array}{l}n \\ L\end{array}\right) z^{L}={ }_{1} F_{0}(-n \mid-z)$ has the generalization

$$
[\operatorname{det}(1+z)]^{n}=\sum_{0<2 L<n}\left(\begin{array}{c}
n \\
2 L
\end{array}\right) \chi^{L}(z)_{2} F_{1}\left(\begin{array}{ll}
2 L-n, & -n-1 \\
2 L+2
\end{array} \mid \Delta(z)\right)
$$

valid for any $2 \times 2$ matrix $z$. Here, $\chi^{L}$ denotes the character of the representation $\rho^{L}$. If $z$ is restricted to lie in the subgroup $S U(2)$, then $(9.1)$ reduces to the Peter-Weyl expansion

$$
[\operatorname{det}(1+u)]^{n}=\sum_{0<2 L<n} a_{L} \chi^{L}(u)
$$


in which the coefficients are given by (A.2) as

$$
a_{L}=\int_{S U(2)}[\operatorname{det}(1+u)]^{n} \chi^{L}(u) d u=\frac{2 L+1}{n+1}\left(\begin{array}{c}
2 n+2 \\
n-2 L
\end{array}\right) .
$$

However, from our point of view (9.1) is the simplest member of a family of much more complicated analogs of the binomial theorem. Indeed, (9.1) can be derived as a special case of the formula

$$
\begin{aligned}
{[\operatorname{det}(z} & +w)]^{n+l_{1}+l_{2}}=\left(2 l_{1}+1\right)^{-1}\left(2 l_{2}+1\right)^{-1} \\
& \times \sum_{x, L} \sum_{\alpha, \beta, \gamma, \delta, \varepsilon, k}(2 L+1)(-1)^{\beta-\gamma+\delta+e} \Delta(z)^{x+l_{2}+L} \Delta(w)^{n-L-l_{1}-x} \\
& \times\left(N^{\lambda_{1}}(n+2)\right)_{\varepsilon, k ; \delta, \delta-\varepsilon+k}\left(N^{\lambda_{2}}(x+2)^{-1}\right)_{\beta, \delta-\varepsilon+k ; \beta-\alpha-\delta+\varepsilon-k,-\alpha} \\
& \times\left(N^{\lambda_{3}}(n-2 L-x+2)^{-1}\right)_{\gamma+\alpha-\beta+\delta+k, \alpha+\delta-\beta+k-\varepsilon ; \varepsilon,-\gamma} \\
& \times D_{-k \alpha}^{l_{2}}\left(\frac{\left(z^{*}+w^{*}\right) z}{\Delta(z+w)^{1 / 2} \Delta(z)^{1 / 2}}\right) D_{\beta \gamma}^{L}\left(\frac{z^{*} w}{\Delta(z)^{1 / 2} \Delta(w)^{1 / 2}}\right) \\
& \times D_{\gamma+\alpha+\delta-\beta+k, \delta}^{l_{1}}\left(\frac{w^{*}(z+w)}{\Delta(w)^{1 / 2} \Delta(z+w)^{1 / 2}}\right)
\end{aligned}
$$

where $\lambda_{1}=\lambda^{0}\left(\cdot ; l_{1}, l_{2}\right), \lambda_{2}=\lambda^{0}\left(\cdot ; l_{2}, L\right)$, and $\lambda_{3}=\lambda^{0}\left(\cdot ; l_{1}, L\right)$; the symbol $N$ denotes the normalized gamma function (Definition 3.2); and matrix entries are relative to Gelfand-Tsetlin bases. This formula holds for all matrices $z$ and $w$ which are positive (scalar) multiples of matrices in $S U(2)$; but since $z^{*}=\Delta(z) z^{-1}$ and $w^{*}=\Delta(w) w^{-1}$, formula (9.4) has an analytic continuation that is valid for all $z, w \in G L(2, C)$. Observe, in analogy to the usual binomial theorem, that in (9.4) two gamma functions "appear in the denominator" and one gamma function "appears in the numerator." One can also derive other analogs of the binomial theorem that are still more complicated than (9.4).

The proofs of results such as these require knowledge of another class of matrix-valued special functions, the generalized Bessel functions for the conformal group. Such Bessel functions arise in terms of the structure of the irreducible unitary representations of the motion group $U(2) \subseteq C^{2 \times 2}$, in complete analogy to the role of classical Bessel functions in the representation theory of the motion group of the plane. Note in particular, that the classical Bessel functions are realized as infinite series each term of which contains two gamma functions in the denominator. The representation property of these classical Bessel functions is closely related to the classical binomial theorem. In the case of the matrix-valued analogs of the Bessel functions, it is possible to obtain series representations in which each summand contains two inverse gamma matrices. The representation property of these new Bessel functions leads to the above analogs of the binomial theorem. These Bessel functions and their properties will be the subject of our subsequent papers [9], [23].

Appendix A: The hypergeometric function. In what follows, we bring together the properties of the hypergeometric functions ${ }_{p} F_{q}$ that are needed for the fine 
structure of $\hat{U}$ and the applications to $\Gamma^{\lambda^{0}}$. A standard reference is [18].

For given nonnegative integers $p$ and $q,{ }_{p} F_{q}$ is defined in terms of numerator parameters $a_{1}, \ldots, a_{p}$, denominator parameters $b_{1}, \ldots, b_{q}$, and argument $z$ by the hypergeometric series

$$
\begin{aligned}
{ }_{p} F_{q}\left(\begin{array}{l}
a_{1} a_{2} \cdots a_{p} \\
b_{1} b_{2} \cdots b_{q}
\end{array} \mid z\right)= & \sum_{n=0}^{\infty} \frac{z^{n}}{n !} \frac{\Gamma\left(a_{1}+n\right)}{\Gamma\left(a_{1}\right)} \frac{\Gamma\left(a_{2}+n\right)}{\Gamma\left(a_{2}\right)} \cdots \frac{\Gamma\left(a_{p}+n\right)}{\Gamma\left(a_{p}\right)} \\
& \times \frac{\Gamma\left(b_{1}\right)}{\Gamma\left(b_{1}+n\right)} \frac{\Gamma\left(b_{2}\right)}{\Gamma\left(b_{2}+n\right)} \cdots \frac{\Gamma\left(b_{q}\right)}{\Gamma\left(b_{q}+n\right)} .
\end{aligned}
$$

Note that whenever a numerator parameter is a nonpositive integer, the series terminates and defines a polynomial in $z$. On the other hand, if a denominator parameter is a nonpositive integer, the coefficients develop poles. However, if a numerator parameter is a larger nonpositive integer, then the series terminates before a pole occurs, and one again obtains a polynomial in $z$.

In our applications, we shall require only the functions ${ }_{p} F_{q}$ with $q=p-1$ and $p=2,3,4,5$ and 7 ; only values of the parameters such that ${ }_{p} F_{q-1}$ is a polynomial in $z$; and with one exception (cf., formula (B.3)), only the argument $z=1$.

The case $p=2$ is classical, ${ }_{2} F_{1}$ being the Gauss hypergeometric function. In this case, Gauss' summation theorem [21, p. 281].

gives the value for unit argument.

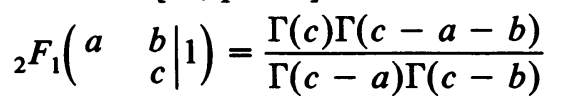

Let $p=3$, assume that $d+e=1+a+b+c$ ("Saalschütz' condition"), and suppose that at least one of the parameters $a, b$, or $c$ is a nonpositive integer so that the ${ }_{3} F_{2}$ series terminates. Then Saalschütz' summation theorem [18, p. 49]

$$
{ }_{3} F_{2}\left(\begin{array}{lll}
a & b & c \\
& d & e
\end{array} \mid 1\right)=\frac{\Gamma(d) \Gamma(1+a-e) \Gamma(1+b-e) \Gamma(1+c-e)}{\Gamma(1-e) \Gamma(d-a) \Gamma(d-b) \Gamma(d-c)}
$$

gives the value for unit argument.

A hypergeometric function is said to be well-poised if $q=p-1$ and $a_{j+1}+b_{j}=$ $a_{1}+1$ for all $j=1,2, \ldots, q$. The summation theorem [18, p. 56] for well-poised ${ }_{5} F_{4}$ of unit argument is

$$
\begin{aligned}
& { }_{5} F_{4}\left(\begin{array}{ccccc}
a & 1+\frac{1}{2} a & b & c & d \\
& \frac{1}{2} a & 1+a-b & 1+a-c & 1+a-d
\end{array}\right) \\
& =\frac{\Gamma(1+a-b) \Gamma(1+a-c) \Gamma(1+a-d) \Gamma(1+a-b-c-d)}{\Gamma(1+a) \Gamma(1+a-b-c) \Gamma(1+a-b-d) \Gamma(1+a-c-d)} .
\end{aligned}
$$

Finally, Dougall's theorem [18, p. 55] gives the summation formula

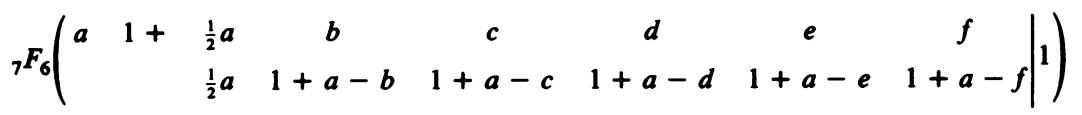

$$
\begin{aligned}
& =\frac{\Gamma(1+a-b) \Gamma(1+a-c) \Gamma(1+a-d) \Gamma(1+a-f) \Gamma(1+a-b-c-d)}{\Gamma(1+a) \Gamma(1+a-b-c) \Gamma(1+a-b-d) \Gamma(1+a-c-d) \Gamma(1+a-b-f)} \\
& \times \frac{\Gamma(1+a-b-c-f) \Gamma(1+a-b-d-f) \Gamma(1+a-c-d-f)}{\Gamma(1+a-c-f) \Gamma(1+a-d-f) \Gamma(1+a-b-c-d-f)}
\end{aligned}
$$


for well-poised ${ }_{7} F_{6}$ of unit argument, provided $1+2 a=b+c+d+e+f$.

Appendix B: Fine structure of the representations of $U(2)$. Underlying the calculations in $\$ 8$ is the explicit detailed knowledge of the properties of the irreducible representations of $U(2)$ (or equivalently, the finite-dimensional holomorphic representations of the complexification $G L(2, \mathrm{C})$ ) under coupling (the Clebsch-Gordan, or Wigner coefficients) and recoupling (the Racah coefficients) as states of angular momentum in quantum mechanics. Thus, in this appendix we list the required formulas, and provide a brief explanation of the concepts for those readers knowledgable in representation theory but unfamiliar with this fine structure. Some of the details can be found in [19], but a more comprehensive treatment such as [1], [2] or [14] is needed.

Recall the exposition at the outset of $\$ 3$ concerning the representations $\rho^{l}$ of $A=G L(2, \mathrm{C})$ and the spaces $V^{l}$ in which they act. There is a natural basis $\left\{e_{m}^{l}\right.$ : $-l<m<l\}$, the Gelfand-Tsetlin basis, for the space $V^{l}$ relative to which the matrix entries of $\rho^{l}$ are given by the Wigner $D$-function. Thus,

$$
\rho^{l}(a) e_{m}^{l}=\sum_{m^{\prime}} D_{m^{\prime} m}^{l}(a) e_{m^{\prime}}^{l}
$$

for $a \in A$ (actually, any $2 \times 2$ matrix $a$, whether or not $\Delta(a) \neq 0$ ) and $-l<m<l$. Although not needed below, we mention that $e_{m}^{l}$ is a scalar multiple of the monomial in $V^{l}$ of degree $l+m$, the scalar being chosen so that the basis is orthonormal relative to an inner product on $V^{l}$ in which the restriction of $\rho^{l}$ to $U$ is unitary. Explicitly, the entries $D_{m^{\prime} m}^{l}$ are the polynomials in the coordinates of $a$ given by [10, (9-71), p. 354] (cf. also [19, (7), p. 116])

$$
\begin{aligned}
D_{m^{\prime} m}^{l}(a)= & \sum_{n=0} \frac{\left[\left(l+m^{\prime}\right) !\left(l-m^{\prime}\right) !(l+m) !(l-m) !\right]^{1 / 2}}{n !\left(l-m^{\prime}-n\right) !(l-m-n) !\left(m^{\prime}+m+n\right) !} \\
& \times\left(a_{12}\right)^{l-m-n}\left(a_{21}\right)^{l-m^{\prime}-n}\left(a_{11}\right)^{m+m^{\prime}+n}\left(a_{22}\right)^{n}
\end{aligned}
$$

where the (finite) limits of summation are determined by the poles of the gamma functions in the denominator. According to (A.1), $D_{m^{\prime} m}^{l}$ is described in terms of ${ }_{2} F_{1}$ as

$$
\begin{aligned}
D_{m^{\prime} m}^{l}(a)= & a_{12}^{l-m} a_{21}^{l-m^{\prime}} a_{11}^{m+m^{\prime}} \frac{1}{\Gamma\left(m^{\prime}+m+1\right)}\left[\frac{\Gamma(l+m+1) \Gamma\left(l+m^{\prime}+1\right)}{\Gamma(l-m+1) \Gamma\left(l-m^{\prime}+1\right)}\right]^{1 / 2} \\
& \times_{2} F_{1}\left(\begin{array}{c}
m-l, m^{\prime}-l \\
m+m^{\prime}+1
\end{array} \mid \frac{a_{11} a_{22}}{a_{12} a_{21}}\right)
\end{aligned}
$$

where we have used the fact that $\Gamma(z+n) \Gamma(z)^{-1}=(-1)^{n} \Gamma(1-z) \Gamma(1-z-n)^{-1}$ for integer $n$.

Next, recall from $\$ 4$ that we are interested in the tensor product representation $\rho^{l_{1}} \otimes \rho^{l_{2}}$ of $A$. Let us refer to this representation as $\pi^{l_{1}, l_{2}}$ and set $W^{l_{1}, l_{2}}=V^{l_{1}} \otimes V^{l_{2}}$. Then the Clebsch-Gordan formula for $A$ gives the multiplicity-free decomposition

$$
\pi^{l_{1}, l_{2}}(a)=\sum_{l=\left|l_{1}-l_{2}\right|}^{l_{1}+l_{2}} \Delta(a)^{l_{1}+l_{2}-l^{l}} \rho^{l}(a)
$$


for $a \in A$. In terms of the underlying representation space,

$$
W^{l_{1}, l_{2}}=\sum_{l=\left|l_{1}-l_{2}\right|}^{l_{1}+l_{2}} V^{l}
$$

and there is a basis, the Clebsch-Gordan basis $\left\{E_{l, m}^{l_{1}, l_{2}}:\left|l_{1}-l_{2}\right|<l<l_{1}+l_{2}\right.$ and $-l<m<l\}$, relative to which the matrix of $\pi^{l_{1}, l_{2}}$ is in block diagonal form. The Clebsch-Gordan transformation $\mathcal{C}: W^{l_{1}, l_{2}} \rightarrow W^{l_{1}, l_{2}}$ takes the basis $\left\{E_{l, m}^{l_{1}, l_{2}}\right\}$ to the tensor product of Gelfand-Tsetlin bases $\left\{e_{m_{1}}^{l_{1}} \otimes e_{m_{2}}^{l_{2}}\right\}$, and the matrix entries $C_{m_{1}, m_{2}, m}^{l_{1}, l_{2}, l}$ of $\mathcal{C}$, defined by

$$
e_{m_{1}}^{l_{1}} \otimes e_{m_{2}}^{l_{2}}=\sum_{l, m}\left(C_{m_{1}, m_{2}, m}^{l_{1}, l_{2}, l}\right) E_{l, m}^{l_{1}, l_{2}}
$$

are known as the Wigner coefficients (or with less justification, Clebsch-Gordan coefficients) for $\rho^{l_{1}} \otimes \rho^{l_{2}}$. In point of fact, for each $l$ on the right side of (B.6) only one coefficient (namely, $m=m_{1}+m_{2}$ ) is nonzero. That is to say,

$$
C_{m_{1}, m_{2}, m}^{l_{1}, l_{2}, l}=0 \text { for } m \neq m_{1}+m_{2}
$$

and

$$
e_{m_{1}}^{l_{1}} \otimes e_{m_{2}}^{l_{2}}=\sum_{l=\left|l_{1}-l_{2}\right|}^{l_{1}+l_{2}}\left(C_{m_{1}, m_{2}, m_{1}+m_{2}}^{l_{1}, l_{2}, l}\right) E_{l, m_{1}+m_{2}}^{l_{1}, l_{2}}
$$

The nonzero Wigner coefficients can be calculated explicitly [19, (10), p. 193], [2, (2.34), p. 34] by means of the $S U(2)$ Lie algebra as

$$
\begin{aligned}
C_{m_{1}, m_{2}, m_{1}+m_{2}}^{l_{1}, l_{2} l}=\left[(2 l+1)\left(l_{1}+m_{1}\right) !\left(l_{1}-m_{1}\right) !\left(l_{2}+m_{2}\right) !\right. \\
\times\left[\frac{\left(l_{1}+l_{2}-l\right) !\left(l_{1}-l_{2}+l\right) !\left(l_{2}-l_{1}+l\right) !}{\left(l_{1}+l_{2}+l+1\right) !}\right]^{1 / 2} \sum_{n=0} \frac{(-1)^{n}}{n !} \\
\quad \times \frac{1}{\left(l_{1}-m_{1}-n\right) !\left(l_{2}+m_{2}-n\right) !\left(l_{1}+l_{2}-l-n\right) !\left(l-l_{1}-m_{2}+n\right) !\left(l-l_{2}+m_{1}+n\right) !}
\end{aligned}
$$

or in terms of the ${ }_{3} F_{2}$ series as

$$
\begin{gathered}
{\left[\frac{(2 l+1)\left(l_{1}+m_{2}\right) !\left(l_{2}-m_{2}\right) !\left(l+m_{1}+m_{2}\right) !\left(l-m_{1}-m_{2}\right) !\left(l_{1}-l_{2}+l\right) !\left(l_{2}-l_{1}+l\right) !}{\left(l_{1}-m_{1}\right) !\left(l_{2}+m_{2}\right) !\left(l_{1}+l_{2}-l\right) !\left(l_{1}+l_{2}+l+1\right) !}\right]^{1 / 2}} \\
\left.\quad \times \frac{1}{\left(l-l_{1}-m_{2}\right) !\left(l-l_{2}+m\right) !}{ }_{3} F_{2}\left(\begin{array}{c}
-l_{1}+m_{1},-l_{2}-m_{2},-l_{1}-l_{2}+l \\
l-l_{1}-m_{2}+1, l-l_{2}+m_{1}+1
\end{array}\right)^{1}\right) .
\end{gathered}
$$


These coefficients possess the following symmetries

$$
\begin{aligned}
C_{m_{1}, m_{2}, m_{1}+m_{2}}^{l_{1}, l_{2}, l} & =(-1)^{l_{1}-m_{1}}\left(\frac{2 l+1}{2 l_{2}+1}\right)^{1 / 2} C_{m_{1},-m_{1}-m_{2},-m_{2}}^{l_{1}, l_{2}} \\
& =(-1)^{l_{2}+m_{2}}\left(\frac{2 l+1}{2 l_{1}+1}\right)^{1 / 2} C_{-m_{1}-m_{2}, m_{2},-m_{1}}^{l, l_{2}, l_{1}} \\
& =(-1)^{l_{1}+l_{2}-l} C_{m_{2}, m_{1}, m_{1}+m_{2}}^{l_{2}, l_{1}, l}=(-1)^{l_{1}+l_{2}-l} C_{-m_{1}, m_{1}, l_{2}, l}^{l_{1},-m_{2},-m_{1}-m_{2}} \\
& =C_{1 / 2\left(l_{1}-l_{2}+m_{1}-m_{2}, 1 / 2\left(l_{1}-l_{2}-m_{1}+m_{2}, l_{1}-l_{2}\right.\right.}^{1 / 2\left(l_{1}+l_{2}+m_{1}+m_{2}\right) 1 / 2\left(l_{1}+l_{2}-m_{1}-m_{2}\right), l}
\end{aligned}
$$

and satisfy the so-called orthogonality and completeness relations (equivalent to the unitarity of the transformation $($ )

$$
\sum_{l=\left|l_{1}-l_{2}\right|}^{l_{1}+l_{2}} C_{m_{1}, m-m_{1}, m}^{l_{1}, l_{2}, l} C_{m_{1}^{\prime}, m-m_{1}^{\prime}, m}^{l_{1}, l_{2}, l}=\delta_{m_{1} m_{1}^{\prime}}
$$

and

$$
\sum_{m_{1}=-l_{1}}^{l_{1}} C_{m_{1}, m-m_{1}, m}^{l_{1}, l_{2}, l} C_{m_{1}, m-m_{1}, m}^{l_{1}, l_{2}, l^{\prime}}=\delta_{l l^{\prime}}
$$

From (B.6) and elementary linear algebra, the matrix entries of $\pi^{l_{1}, l_{2}}$ relative to the above two bases for $W^{l_{1}, l_{2}}$ are related by the formula

$$
\left(\pi^{l_{1}, l_{2}}(a)\right)_{e^{l_{1} \otimes e^{l_{2}}}}=C^{*} \cdot\left(\pi^{l_{1}, l_{2}}(a)\right)_{E^{l_{1}, l_{2}}} \cdot \mathrm{C} .
$$

Equivalently,

$$
D_{m_{1}^{\prime} m_{1}}^{l_{1}}(a) D_{m_{2}^{\prime} m_{2}}^{l_{2}}(a)=\sum_{l=\left|l_{1}-l_{2}\right|}^{l_{1}+l_{2}} C_{m_{1}^{\prime}, m_{2}^{\prime}, m_{1}^{\prime}+m_{2}^{\prime}}^{l_{1}, l_{2}, l} \Delta(a)^{l_{1}+l_{2}-l} D_{m_{1}^{\prime}+m_{2}^{\prime}, m_{1}+m_{2}}^{l}(a) C_{m_{1}, m_{2}, m_{1}+m_{2}}^{l_{1}, l_{2}, l}
$$

or by inversion (i.e., (B.13)),

$$
\Delta(a)^{l_{1}+l_{2}-l} D_{m^{\prime}, m}^{l}(a)=\sum_{m_{1}^{\prime}, m_{1}} C_{m_{1}^{\prime}, m^{\prime}-m_{1}^{\prime}, m^{\prime}}^{l_{1} l_{2}, l} D_{m_{1}^{\prime} m_{1}}^{l_{1}}(a) D_{m^{\prime}-m_{1}^{\prime}, m-m_{1}}^{l_{2}}(a) C_{m_{1}, m^{\prime}-m_{1}, m}^{l_{1}, l_{2}, l}
$$

From the physical point of view, the Wigner coefficients "couple" two states of angular momentum into a third. We shall also need the recoupling or Racah coefficients which relate two different bases for the tensor product of three representations. Thus, fix parameters $l_{1}, l_{2}$, and $l_{3}$, and set $W^{l_{1}, l_{2}, l_{3}}=V^{l_{1}} \otimes V^{l_{2}} \otimes V^{l_{3}}$. Associated one way, we have by (B.5)

$$
W^{l_{1}, l_{2}, l_{3}}=\sum_{l=\left|l_{1}-l_{2}\right|}^{l_{1}+l_{2}}\left(V^{l} \otimes V^{l_{3}}\right)
$$

whereas another way

$$
W^{l_{1}, l_{2}, l_{3}}=\sum_{l^{\prime}=\left|l_{2}-l_{3}\right|}^{l_{2}+l_{3}}\left(V^{l_{1}} \otimes V^{l^{\prime}}\right)
$$




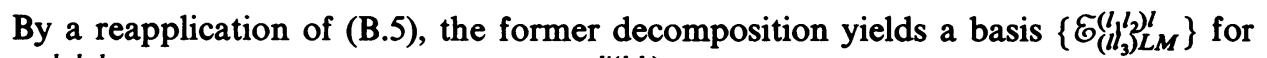
$W^{l_{1}, l_{2}, l_{3}}$, and the latter yields a basis $\left\{\mathcal{E}_{\left(l_{1} l_{2} l_{3}\right) L M}^{l^{\prime}\left(l_{2}\right)}\right.$. Here, $L$ indexes the representations that actually appear (in general, with multiplicity) in $\rho^{l_{1}} \otimes \rho^{l_{2}} \otimes \rho^{l_{3}} ;-L<M<L$; and $l, l^{\prime}$ are multiplicity indices. The Racah transformation $\Re: W^{l_{1}, l_{2}, l_{3}} \rightarrow W^{l_{1}, l_{2}, l_{3}}$ takes the latter basis into the former basis. The matrix entries $R_{l_{3}}^{l_{1} l_{2}^{l}}$, are independent of $M$ and defined by

$$
\mathcal{E}_{\left(l, l_{3}\right) L M}^{\left(l_{1}, l_{2}\right) l}=\sum_{l^{\prime}} R_{l_{3} L^{\prime}}^{l_{1} l_{2} l} \mathcal{E}_{\left(l_{1} l^{\prime}\right) L M}^{l^{\prime}\left(l^{l_{3}} l_{3}\right)}
$$

For reasons of symmetry, the Racah coefficients or $6-j$ symbols

$$
\left\{\begin{array}{l}
l_{1} l_{2} l \\
l_{3} L l^{\prime}
\end{array}\right\}
$$

are defined by the formula

$$
R_{l_{3} L^{\prime}}^{l_{1} l_{2} l^{\prime}}=\left\{\begin{array}{c}
l_{1} l_{2} l \\
l_{3} L l^{\prime}
\end{array}\right\}(-1)^{2 L}\left[(2 l+1)\left(2 l^{\prime}+1\right)\right]^{1 / 2} .
$$

From (B.18), (B.19), and the relations (B.12) and (B.13), one can derive the following (essentially defining) relations for the $6-j$ symbols,

$$
\begin{gathered}
\sum_{L}(-1)^{2 l}\left[(2 L+1)\left(2 l^{\prime}+1\right)\right]^{1 / 2}\left\{\begin{array}{c}
l_{1} l_{2} L \\
l_{3} l l^{\prime}
\end{array}\right\} C_{m_{1}, m_{2}, m_{1}+m_{2}}^{l_{1}, l_{2}, L} C_{m_{1}+m_{2}, m_{3}, m_{1}+m_{2}+m_{3}}^{L, l_{3}, l} \\
=C_{m_{3}, m_{2}, m_{3}+m_{2}}^{l_{3}, l_{2}, l^{\prime}} C_{m_{3}+m_{2}, m_{1}, m_{1}+m_{2}+m_{3} .}^{l^{\prime}, l_{1}, l}
\end{gathered}
$$

The $6-j$ symbols are symmetric under permutations of columns and under the inversion of any two columns.

In $\S 8$, we use the explicit form [14, p. 140] of one $6-j$ symbol; viz.,

$$
\begin{aligned}
\left\{\begin{array}{c}
l_{2} l_{1} L \\
l_{2} l_{1} l
\end{array}\right\}= & (-1)^{2 l_{2}+2 l_{1}}\left[\frac{\left(L+l_{2}-l_{1}\right) !\left(l_{1}+l_{2}+L+1\right) !\left(l_{1}+l_{2}-L\right) !\left(l+l_{1}-l_{2}\right) !}{\left(l+l_{2}-l_{1}\right) !\left(l_{1}+l_{2}+l+1\right) !\left(l_{1}+l_{2}-l\right) !\left(L+l_{1}-l_{2}\right) !}\right] \\
& \times \sum_{n}(-1)^{n} \frac{\left(L+l_{1}-l_{2}+n\right) !\left(L+l_{1}-l_{2}+n\right) !\left(l+2 l_{2}-L-n\right) !}{n !\left(l_{1}+l_{2}-L-n\right) !\left(l_{1}+l_{2}-L-n\right) !\left(l-2 l_{2}+L+n\right) !(2 L+1+n) !} .
\end{aligned}
$$

For details concerning the Wigner and Racah coefficients, see [2, Chapter III]; for example, the most general $6-j$ symbol appears as formula (3.15) on p. 43.

Addendum: The gamma function for $U(n, n)$. We shall explain how the results in $\$ \$ 3$ and 4 relating to the evaluation of the eigenvalues of the gamma function carry over from the conformal group $U(2,2)$ to the groups $U(n, n)$ for general $n$.

The group $U(n, n)$ is defined as the collection of all $2 n \times 2 n$ complex matrices $g$ such that $g p g^{t}=p$, in complete analogy to the case $n=2$ which corresponds to the conformal group (cf. formula (2.2) et seq.-here the entries $g_{i j}$ and $p_{i j}$ are $n \times n$ matrices). Throughout this addendum $G=U(n, n)$. Now, the maximal compact subgroup $K$ of $G$ is isomorphic to $U(n) \times U(n)$ and $P$ is the cone of positive-definite $n \times n$ Hermitian matrices. The group $A=G L(n, C)$ acts on $P$ by $r \mapsto a^{*} r a$ and the measure $d_{*} r=(\operatorname{det} r)^{-n} d r$ is $A$-invariant. Then equation (3.11) defines the 
generalized gamma function for $U(n, n)$ of weight $\lambda^{0}$. Let us describe the representations $\lambda^{0}$ of $\mathcal{Q}=A \times A$ that appear.

An $n$-tuple $[m]=\left(m_{1}, m_{2}, \ldots, m_{n}\right)$ of integers such that $m_{1}>m_{2}>\cdots>m_{n}$ is called a highest weight. To each highest weight $[m]$ there corresponds a unique (equivalence class of) irreducible finite-dimensional rational representation $\rho_{[\mathrm{m}]}$ of $A$, different highest weights correspond to inequivalent representations, and these are all the irreducible finite-dimensional holomorphic representations of $A$ (cf. [7]). The restrictions of these representations to the subgroup $U=U(n)$ exhaust the dual object $\hat{U}$. Let us denote by $\{m\}$ a highest weight such that $m_{n}=0$, so $\{m\}=\left(m_{1}, \ldots, m_{n-1}, 0\right)$ with the integers $m_{j}$ nonincreasing and nonnegative. Then for the general highest weight $[m], \rho_{[m]}(a)=\Delta(a)^{m_{n}} \rho_{\left\{m^{\prime}\right\}}(a)$ for all $a$ in $A$, where $\left\{m^{\prime}\right\}=\left(m_{1}-m_{n}, \ldots, m_{n-1}-m_{n}, 0\right)$. The restrictions to $U_{0}=S U(n)$ of the representations $\rho_{\{m\}}$ exhaust the dual $\hat{U}_{0}$. Let $\hat{\mathbb{Q}}_{0}$ denote the collection of representations $\lambda^{0}$ of $\mathcal{Q}$ of the form

$$
\lambda^{0}\left(a_{1}, a_{2}\right)=\rho_{\{m\}}\left(a_{1}\right) \otimes \rho_{\{q\}}\left(a_{2}\right)
$$

for $a_{1}$ and $a_{2}$ in $A$. These are the "weights" $\lambda^{0}$ that appear in the definition of the gamma function for $U(n, n)$ (cf. equation (3.4) et seq.). To be completely clear in the display of parameters we can set

$$
\lambda^{0}=\lambda^{0}(\cdot ;\{m\},\{q\})=\lambda^{0}\left(\cdot ; m_{1}, \ldots, m_{n-1} ; q_{1}, \ldots, q_{n-1}\right) .
$$

In this context (i.e., of $U(n, n)$ ), Theorem 3.1 and Corollary 3.3 are valid as stated, except that now $\operatorname{Re}(\alpha)>n-1$. However, in order to generalize to the present context the results of $\S 4$ for the evaluation of the eigenvalues of $\Gamma^{\lambda^{0}}(\alpha)$, we shall assume that one or the other of the representations $\rho_{\{m\}}$ or $\rho_{\{q\}}$ is totally symmetric. By this we mean that the highest weight, say $\{q\}$, is of the form

$$
\{q\}=\dot{q}=(q, 0,0, \ldots, 0) \text {. }
$$

This condition is equivalent to the statement that the representation $\rho_{\dot{q}}$ has a "Young diagram" with just one row, or alternatively that the restriction of $\rho_{\dot{q}}$ to $S U(n)$ appears in the decomposition of $L^{2}(S U(n) / S U(n-1))$. But from our point of view the important feature of the representations $\rho_{\dot{q}}$ lies in the fact that the representations $u \mapsto \lambda^{0}\left(u, \bar{u} ; m_{1}, \ldots, m_{n-1} ; q, 0, \ldots, 0\right)$ of $U=U(n)$ are all multiplicity free. Indeed,

$$
\lambda^{0}(u, \bar{u})=\sum_{[M]} \bigoplus_{[M]}(u)
$$

where the sum is over all highest weights $[M]=\left(M_{1}, \ldots, M_{n}\right)$ such that

$$
\sum_{j=1}^{n} M_{j}=q+\sum_{j=1}^{n-1} m_{j} .
$$

Thus, as before, the commutation relation (3.12) and the Clebsch-Gordan formula (ADD-3) imply a spectral decomposition

$$
\Gamma^{\lambda^{0}}(\alpha)=\sum \bigoplus_{[M]} \gamma_{M}^{\lambda^{0}}(\alpha) I_{M}
$$


of the gamma function $\Gamma^{\lambda^{0}}$, where the highest weights $[M]$ satisfy (ADD-4) and $I_{M}$ denotes the identity operator on the space of $\rho_{[M]}$.

The technical calculations in $\$ 8$ can be extended to the case at hand, although one is required to break new ground in the theory of multidimensional generalized hypergeometric functions [22], and the conceptual result of (ADD-5) can be made explicit by the calculation of the eigenvalues $\gamma_{M}^{\lambda^{0}}(\alpha)$. Here, we will be satisfied with a statement of the results, and shall leave the details of proofs and applications to a subsequent article.

Theorem. Let $\lambda^{0} \in \hat{\mathfrak{Q}}_{0}$ be given by (ADD-1) and $\dot{q}$ by (ADD-2). Then the eigenvalues of the gamma function $\Gamma^{\lambda^{0}}(\alpha)$ are

$$
\begin{aligned}
\gamma_{M}^{\lambda^{0}}(\alpha)= & \Gamma\left(\alpha+m_{1}\right) \Gamma\left(\alpha+m_{2}-1\right) \Gamma\left(\alpha+m_{3}-2\right) \cdots \Gamma(\alpha-n+1) \\
& \times \Gamma\left(\alpha+m_{1}+q+n-1\right) \Gamma\left(\alpha+m_{2}+q+n-2\right) \cdots \Gamma(\alpha+q) \\
& \times \Gamma\left(\alpha+M_{1}+n-1\right)^{-1} \Gamma\left(\alpha+M_{2}+n-2\right)^{-1} \cdots \Gamma\left(\alpha+M_{n}\right)^{-1}
\end{aligned}
$$

(ADD-6)

where the highest weights $[M]$ are constrained by (ADD-4).

We shall say that the gamma function $\Gamma^{\lambda^{0}}$ is multiplicity-free if its weight $\lambda^{0}$ has the property that $u \mapsto \lambda^{0}(u, \bar{u})$ is a multiplicity-free representation of $U$. In addition to the case treated in the theorem, the gamma function for $U(n, n)$ is multiplicityfree when $\rho_{\{q\}}$ (or equivalently $\rho_{\{m\}}$ ) is conjugate to a totally symmetric representation; i.e., when $\{q\}=(q, \ldots, q, 0)$, and the eigenvalues of the gamma matrix can be found by similar methods in this case also.

\section{REFERENCES}

1. L. C. Biedenharn and H. Van Dam, Editors, Quantum theory of angular momentum, Academic Press, New York, 1965.

2. D. M. Brink and G. R. Satchler, Angular momentum, Clarendon Press, Oxford, 1962.

3. S. Gelbart, Bessel functions, representation theory, and automorphic functions, Proc. Sympos. Pure Math., vol. 26, Amer. Math. Soc., Providence, R. I., 1973, pp. 343-345.

4. R. Godement, Fonctions automorphes, Séminaire Cartan, University of Paris, 1957-1958.

5. K. I. Gross and R. A. Kunze, Fourier-Bessel transforms and holomorphic discrete series, Conference on Harmonic Analysis, Lecture Notes in Math., vol. 266, Springer-Verlag, Berlin and New York, 1972, pp. 79-122.

6. Generalized Bessel transforms and unitary representations, Proc. Sympos. Pure Math., vol. 26, Amer. Math. Soc., Providence, R. I., 1973, pp. 347-350.

7. Bessel functions and representation theory, II: Holomorphic discrete series and metaplectic representations, J. Functional Anal. 25 (1977), 1-49.

8. K. I. Gross, W. J. Holman III and R. A. Kunze, The generalized gamma function, new Hardy spaces, and representations of holomorphic type for the conformal group, Bull. Amer. Math. Soc. 83 (1977), 412-415.

9. K. I. Gross and W. J. Holman III, Matrix-valued special functions and representation theory of the conformal group. II: The generalized Bessel functions (in preparation).

10. M. Hammermesh, Group theory and its application to physical problems, Addison-Wesley, Reading, Mass., 1964.

11. Harish-Chandra, Representations of semi-simple Lie groups. IV, V, VI, Amer. J. Math. 77 (1955), 743-777; 78 (1956), 1-41; 78 (1956), 564-628.

12. L. K. Hua, Harmonic analysis of functions of several complex variables in the classical domains, Transl. Math. Mono., vol. 6, Amer. Math. Soc., Providence, R. I., 1963. 
13. H. Jakobsen and $M$. Vergne, Wave and Dirac operators and representations of the conformal group, J. Functional Anal. 24 (1977), 52-106.

14. A. P. Jucys and A. A. Bandzaitis, Theoriya momenta kolichestva doizheniya v kvantovoi mechanike, "Mintis", Vilnius, 1965.

15. A. Knapp and K. Okamoto, Limits of holomorphic discrete series, J. Functional Anal. 9 (1972), 375-409.

16. H. Rossi and M. Vergne, Representations of certain solvable Lie groups on Hilbert spaces of holomorphic functions and the application to the holomorphic discrete series of a semi-simple Lie group, $\mathrm{J}$. Functional Anal. 13 (1973), 324-389.

17. Analytic continuation of the holomorphic discrete series of a semi-simple Lie group, Acta Math. 136 (1976), 1-59.

18. L. J. Slater, Generalized hypergeometric functions, Cambridge Univ. Press, New York, 1966.

19. N. J. Vilenkin, Special functions and the theory of group representations, Amer. Math. Soc. Transl., Amer. Math. Soc., Providence, R. I., 1968.

20. N. Wallach, Analytic continuation for the holomorphic discrete series. I, II, Trans. Amer. Math. Soc. 251 (1979), 1-17; 19-37.

21. E. T. Whittaker and G. N. Watson, A course of modern analysis, 4th ed., Cambridge Univ. Press, New York, 1927.

22. W. J. Holman III, Summation theorems for hypergeometric series in U(n), SIAM J. Math. Anal. (to appear).

23. Phys. (to appear).

Department of Mathematics, University of North Carolna, Chapel Hill, North Carolina 27514 (Current address of K. I. Gross)

Department of Mathematics, University of the South, Sewaneg, Tennessee 37375

Current address (W. J. Holman III): Bell Laboratories, Inc., Indian Hill, Naperville, Illinois 60540 